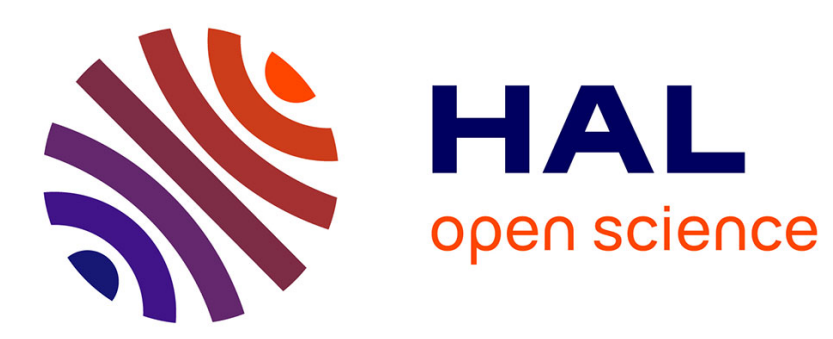

\title{
On the cost of observability in small times for the one-dimensional heat equation
}

\author{
Jérémi Dardé, Sylvain Ervedoza
}

\section{To cite this version:}

Jérémi Dardé, Sylvain Ervedoza. On the cost of observability in small times for the one-dimensional heat equation. Analysis \& PDE, 2019, 10.2140/apde.2019.12.1455 . hal-01619211

\section{HAL Id: hal-01619211 \\ https://hal.science/hal-01619211}

Submitted on 23 Oct 2017

HAL is a multi-disciplinary open access archive for the deposit and dissemination of scientific research documents, whether they are published or not. The documents may come from teaching and research institutions in France or abroad, or from public or private research centers.
L'archive ouverte pluridisciplinaire HAL, est destinée au dépôt et à la diffusion de documents scientifiques de niveau recherche, publiés ou non, émanant des établissements d'enseignement et de recherche français ou étrangers, des laboratoires publics ou privés. 


\title{
On the cost of observability in small times for the one-dimensional heat equation
}

\author{
Jérémi Dardé* Sylvain Ervedoza ${ }^{\dagger}$ \\ Institut de Mathématiques de Toulouse ; UMR 5219 ; Université de Toulouse ; CNRS ; \\ UPS IMT F-31062 Toulouse Cedex 9, France
}

October 18, 2017

\begin{abstract}
In this article, we aim at presenting a new estimate on the cost of observability in small times of the one-dimensional heat equation, which also provides a new proof of observability for the one-dimensional heat equation. Our proof combines several tools. First, it uses a Carleman type estimate borrowed from [6], in which the weight function is derived from the heat kernel and which is therefore particularly easy. We also use explicit computations in the Fourier domain to compute the high-frequency part of the solution in terms of the observations. Finally, we use the Phragmén Lindelöf principle to estimate the low frequency part of the solution. This last step is done carefully with precise estimations coming from conformal mappings.
\end{abstract}

\section{Introduction}

Setting. The goal of this work is to analyze the cost of observability in small times of the one-dimensional heat equation. To fix the ideas, let $L, T>0$ and consider the following heat equation, set in the bounded interval $(-L, L)$ and among some time interval $(0, T)$ :

$$
\begin{cases}\partial_{t} u-\partial_{x}^{2} u=0 & \text { in }(0, T) \times(-L, L), \\ u(t,-L)=u(t, L)=0 & \text { in }(0, T), \\ u(0, x)=u_{0}(x) & \text { in }(-L, L) .\end{cases}
$$

In (1.1), the state $u=u(t, x)$ satisfies a heat equation, with an initial datum $u_{0} \in H_{0}^{1}(-L, L)$.

Our main goal is to study the cost of observability in small times $T$ of the problem (1.1) observed from both sides $x=-L$ and $x=+L$. To be more precise, let us recall that it is by now well-known that there exists $C_{0}(T, L)$ such that all solution $u$ of 1.1 with initial datum $u_{0} \in H_{0}^{1}(-L, L)$ satisfies:

$$
\|u(T)\|_{L^{2}(-L, L)} \leqslant C_{0}(T, L)\left(\left\|\partial_{x} u(t,-L)\right\|_{L^{2}(0, T)}+\left\|\partial_{x} u(t, L)\right\|_{L^{2}(0, T)}\right) .
$$

In fact, the existence of the constant $C_{0}(T, L)$ is a consequence of the null controllability results in small times obtained by [10, [12] in the one-dimensional case. From now on, we denote by $C_{0}(T, L)$ the best constant in the observability inequality 1.2

A precise description of the constant $C_{0}(T, L)$ as $T \rightarrow 0$ is still missing, despite several contributions in this direction, which we would like to briefly recall here. First, the article [36] showed that

$$
\limsup _{T \rightarrow 0} T \log C_{0}(T, L)<\infty
$$

*e-mail: jeremi.darde@math.univ-toulouse.fr.

$\dagger$ †-mail: sylvain.ervedoza@math.univ-toulouse.fr. 
while 19] proved that

$$
\liminf _{T \rightarrow 0} T \log C_{0}(T, L)>0 .
$$

Besides, due to the scaling of the equation, $C_{0}(T, L)$ depends only on the ratio $L^{2} / T$. Therefore, the quantity $T \log C_{0}(T, L)$ should be compared to $L^{2}$. We list below several contributions.

$$
\begin{aligned}
\liminf _{T \rightarrow 0} T \log C_{0}(T, L) & \geqslant \frac{L^{2}}{4}, \\
\liminf _{T \rightarrow 0} T \log C_{0}(T, L) & \geqslant \frac{L^{2}}{2}, \\
\limsup _{T \rightarrow 0} T \log C_{0}(T, L) & \leqslant 2\left(\frac{36}{37}\right)^{2} L^{2}, \\
\limsup _{T \rightarrow 0} T \log C_{0}(T, L) & \leqslant \frac{3^{+} L^{2}}{4},
\end{aligned}
$$

where the notation + in the last estimate means that "any strictly larger number is convenient".

Main result. Our contribution comes in this context. Namely we prove the following result:

Theorem 1.1. Setting

$$
K_{0}=\frac{1}{4}+\frac{\Gamma(1 / 4)^{2}}{8 \sqrt{2} \pi^{2}} \sum_{n \in \mathbb{N}} \frac{(-1)^{n}}{(2 n+1)} \frac{\Gamma(n+1 / 4)}{\Gamma(n+7 / 4)}, \quad\left(K_{0} \simeq 0.6966\right),
$$

where $\Gamma$ denotes the gamma function, for any $K>K_{0}$, we have

$$
\limsup _{T \rightarrow 0} T \log C_{0}(T, L) \leqslant K L^{2}
$$

In fact, there exists a constant $C>0$ such for all $T \in(0,1]$, for all solutions $u$ of 1.1 with initial datum $u_{0} \in H_{0}^{1}(-L, L)$,

$$
\left\|u(T) \exp \left(\frac{x^{2}}{4 T}\right)\right\|_{L^{2}(-L, L)} \leqslant C \exp \left(\frac{K L^{2}}{T}\right)\left(\left\|\partial_{x} u(t,-L)\right\|_{L^{2}(0, T)}+\left\|\partial_{x} u(t, L)\right\|_{L^{2}(0, T)}\right) .
$$

Remark 1.2. The constant $K_{0}$ in (1.5) can alternatively be written as

$$
K_{0}=\frac{1}{4}+\frac{2}{\pi} \frac{\int_{0}^{\frac{\pi}{2}} \ln \left(\cot \left(\frac{t}{2}\right)\right) \sqrt{\cos (t)} d t}{\int_{0}^{\frac{\pi}{2}} \sqrt{\cos (t)} d t},
$$

see Proposition 2.3 in Section 2.

Theorem 1.1 slightly improves the cost of observability in small times when compared to [37]. However, we do not claim that this bound is sharp, and this remains, to our knowledge, an open problem. In particular, we shall comment in Section 4.6 a possible path to improve the estimates given in Theorem 1.1 .

In fact, we believe that Theorem 1.1 is interesting mostly by its proof, presented in Section 2 which combines several arguments. In particular, it uses a Carleman type estimate, which was already used in 6] to derive a good description of the reachable set for the one-dimensional heat equation in terms of domains of holomorphic extension of the states. This Carleman type estimate is used to reduce the problem of observability to an estimate of the low frequency part of the solution of 1.1 . Then, we shall use Fourier analysis on the conjugated heat equation to get an exact formula for the high-frequency part of the solution of 1.1 in terms of the observations. The last part of the argument is a complex analysis argument based on the Phragmén Lindelöf principle. We refer to Sections 2 and 3 for the detailed proof of Theorem 1.1. Let us also mention that Theorem 1.1 is strongly connected to control theory. Indeed, let us consider the 
following null-controllability problem: Given $T>0$ and $y_{0} \in L^{2}(-L, L)$, find control functions $v_{-}, v_{+} \in$ $L^{2}(0, T)$ such that the solution $y$ of

$$
\begin{cases}\partial_{t} y-\partial_{x}^{2} y=0 & \text { in }(0, T) \times(-L, L), \\ y(t,-L)=v_{-}(t) & \text { in }(0, T), \\ y(t,+L)=v_{+}(t) & \text { in }(0, T) \\ y(0, x)=y_{0}(x) & \text { in }(-L, L),\end{cases}
$$

satisfies

$$
y(T, x)=0 \quad \text { in }(-L, L) .
$$

It is well-known (see e.g. [10] or [12]) that for any $T>0$, one can find controls $v_{-}, v_{+}$of minimal $\left(L^{2}(0, T)\right)^{2}$ norm, depending linearly on $y_{0} \in L^{2}(-L, L)$, such that the controlled trajectory, i.e. the solution of 1.9), satisfies 1.10. Besides, the $\mathscr{L}\left(L^{2}(-L, L) ;\left(L^{2}(0, T)\right)^{2}\right)$-norm of the linear map $y_{0} \mapsto\left(v_{-}, v_{+}\right)$is precisely $C_{0}(T, L)$. In other words, $C_{0}(T, L)$ also characterizes the cost of controllability for the one-dimensional heat equation.

We emphasize that Theorem 1.1 also allows to tackle some multi-dimensional settings. Namely, as a consequence of Theorem 1.1 and the control transmutation method (see 34]), one gets the following corollary:

Corollary 1.3. Let $\Omega$ be a smooth bounded domain of $\mathbb{R}^{d}$, and let $\Gamma_{0}$ be an open subset of $\partial \Omega$. Let $a=a(x) \in L^{\infty}\left(\Omega ; M_{d}(\mathbb{R})\right)$ and $\rho \in L^{\infty}(\Omega ; \mathbb{R})$ be such that there exist strictly positive numbers $\rho_{-}, \rho_{+}, a_{-}$ and $a_{+}$such that for all $x \in \Omega$ and $\xi \in \mathbb{R}^{d}$,

$$
a_{-}|\xi|^{2} \leqslant a(x) \xi \cdot \xi \leqslant a_{+}|\xi|^{2}, \quad \rho_{-} \leqslant \rho(x) \leqslant \rho_{+} .
$$

Further assume that there exist a time $S_{0}>0$ and a constant $C>0$ such that for any $\left(w_{0}, w_{1}\right) \in H_{0}^{1}(\Omega) \times$ $L^{2}(\Omega)$, the solution $w$ of

$$
\begin{cases}\rho(x) \partial_{s s} w-\operatorname{div}(a(x) \nabla w)=0 & \text { in }(0, S) \times \Omega \\ w(s, x)=0 & \text { on }(0, S) \times \partial \Omega \\ \left(w(0, x), \partial_{s} w(0, x)\right)=\left(w_{0}(x), w_{1}(x)\right) & \text { in } \Omega\end{cases}
$$

satisfies

$$
\left\|\left(w_{0}, w_{1}\right)\right\|_{H_{0}^{1}(\Omega) \times L^{2}(\Omega)} \leqslant C\|a(x) \nabla w \cdot n\|_{L^{2}\left(\left(0, S_{0}\right) \times \Gamma_{0}\right)} .
$$

We define $C_{0}\left(T, \Omega, \Gamma_{0}\right)$ as the best constant in the following observability inequality: for all $u_{0} \in H_{0}^{1}(M)$, the solution $u$ of

$$
\begin{cases}\rho(x) \partial_{t} u-\operatorname{div}(a(x) \nabla u)=0 & \text { in }(0, T) \times \Omega, \\ u(t, x)=0 & \text { on }(0, T) \times \partial \Omega, \\ u(0, x)=u_{0}(x) & \text { in } \Omega\end{cases}
$$

satisfies

$$
\|u(T)\|_{L^{2}(M)} \leqslant C_{0}\left(T, \Omega, \Gamma_{0}\right)\|a(x) \nabla u \cdot n\|_{L^{2}\left((0, T) \times \Gamma_{0}\right)} .
$$

Then we have, for any $K>K_{0}$,

$$
\limsup _{T \rightarrow 0} T \log C_{0}\left(T, \Omega, \Gamma_{0}\right) \leqslant K S_{0}^{2}
$$

Corollary 1.3 uses the transmutation method and therefore the observability of the corresponding wave equation (1.11), which has been well-studied in the literature. In particular, if the coefficients $\rho$ and $a$ are $C^{2}(\bar{\Omega})$, according to $[1,2,3$, the wave equation $(1.11)$ satisfies the observability inequality 1.12 if and only if all the rays of Geometric Optics meet $\Gamma_{0}$ in a non-diffractive point in time less than $S_{0}$. In case of coefficients $\rho$ and $a$ which are less regular, let us quote the recent works [11 in the one-dimensional case with $\rho$ and $a$ in the Zygmund class, and [7] in the multi-dimensional case for coefficients $\rho \in C^{0}(\bar{\Omega})$ and $a=1$, with $\rho$ satisfying a multiplier type condition similar to the one in [22, 27] in the sense of distributions (and $\rho$ locally $C^{1}$ close to the boundary, see [7, Section 4.2]).

Let us emphasize that Corollary 1.3 can be applied in the one-dimensional case as well for coefficients in the Zygmund class [11. But even in the case $\Omega=(-L, L), \Gamma_{0}=\{-L, L\}, \rho(x)=1, a(x)=1$, we get $S_{0}=2 L$ and thus we obtain an estimate on the cost of observability of the form

$$
\limsup _{T \rightarrow 0} T \log C_{0}(T,(-L, L),\{-L, L\}) \leqslant 4 K_{0}^{+} L^{2},
$$


instead of 1.6. In other words, we have a loss of a factor 4. Therefore, we shall also explain how Theorem 1.1 can be extended to a multi-dimensional case directly when the observation is performed on the whole boundary, see Theorems $4.1,4.2$.

Let us mention that the proofs of the observability inequality of the heat equation for general smooth bounded domains $\Omega$ and observation in an open subset $\Gamma_{0}$ of the boundary in [14, 25] yields that

$$
\limsup _{T \rightarrow 0} T \log C_{0}\left(T, \Omega, \Gamma_{0}\right)<\infty
$$

while on the other hand, 32 proves

$$
\liminf _{T \rightarrow 0} T \log C_{0}\left(T, \Omega, \Gamma_{0}\right) \geqslant \frac{\sup _{\Omega} d\left(x, \Gamma_{0}\right)^{2}}{4} .
$$

To our knowledge, getting more intrinsic geometric upper estimates on the cost of observability in small times in such general settings is still out of reach. However, in geometrical cases which can be obtained by tensorization, some estimates can be obtained, see 33 and Section 4.2 for more details.

We shall also mention that estimating the observability constant in small times for the heat equation in the one-dimensional case is related to the uniform controllability of viscous approximations of the transport equation, see 4, 17, 28, 29]. We refer in particular to Section 4.7 for a more precise discussion on this problem. In particular, the proof in 28, when combined with Theorem 1.1. easily yields an improvement of the results known on this problem, see Section 4.7 and Theorem 4.9 for more details.

As we have seen in the above discussion, there are still some open questions on the observability of the onedimensional constant coefficients parabolic equations, despite the efficiency and robustness of the approach based on Carleman estimates 14, 25. This has justified the development of new manners to derive controllability of parabolic equations, and we shall in particular quote the flatness method developed in [30, 31], a heat packet decomposition [16] or the backstepping approach [5]. Our method comes in this context and provides what seems to be another approach to obtain observability results for the heat equation.

Outline. Section 2 presents the main strategy of the proof of Theorem 1.1 using several technical results that will be proved afterwards, in Section 3 for the ones using new arguments, in Section A for a Carleman type estimate (Theorem 2.1) which can be found also in 6] in a slightly different form. Section 4 provides several comments on Theorem 2.1 and its generalization, including a discussion on what can be done in the multi-dimensional setting in Section 4.1. when the geometry has a tensorized form in Section 4.2 or when the observation is on one side of the domain (Section 4.3) or on some distributed open subset (Section 4.4. We also present in Section 4.5 an alternative proof of a weaker version of Theorem 1.1 based on the uncertainty principles of Landau and Pollack [24] and the result in [13], recovering the result of [37. This led us to discuss the possibility of improving the estimate of the cost of observability in small times in Theorem 1.1 by using a better bound than the one provided by the use of Phragmén Lindelöf principle for entire functions, see Section 4.6 for more details. We end up in Section 4.7 by giving a consequence of our result on the problem of uniform controllability of viscous approximations of transport equations. Section A gives the detailed proof of a rather easy Carleman estimate which is one of the building blocks of our analysis.

Acknowledgments. The authors wish to express their gratitude to Karine Beauchard for stimulating discussions on this work and several comments on a preliminary version of this article.

\section{Proof of Theorem 1.1: main steps}

As said in the introduction, the proof of Theorem 1.1 relies on several steps.

The first step is the following Carleman type estimate.

Theorem 2.1. For any smooth solution $u$ of 1.1 , setting

$$
z(t, x)=u(t, x) \exp \left(\frac{x^{2}-L^{2}}{4 t}\right), \quad(t, x) \in(0, T) \times(-L, L),
$$


we have the inequality:

$$
\int_{-L}^{L}\left|\partial_{x} z(T, x)\right|^{2} d x-\frac{L^{2}}{4 T^{2}} \int_{-L}^{L}|z(T, x)|^{2} d x \leqslant \frac{L}{T^{2}} \int_{0}^{T} t\left(\left|\partial_{x} u(t,-L)\right|^{2}+\left|\partial_{x} u(t, L)\right|^{2}\right) d t .
$$

Theorem 2.1 is based on the study of the equation satisfied by $z$ in 2.1. As $u$ satisfies the heat equation (1.1), the function $z$ in 2.1 satisfies the following equation:

$$
\begin{cases}\partial_{t} z+\frac{x}{t} \partial_{x} z+\frac{1}{2 t} z-\partial_{x}^{2} z-\frac{L^{2}}{4 t^{2}} z=0, & (t, x) \in(0, \infty) \times(-L, L), \\ z(t,-L)=z(t, L)=0, & t \in(0, \infty), \\ z(0, x)=0, & x \in(-L, L) .\end{cases}
$$

One can therefore perform energy estimates on (2.3), which will eventually lead to 2.2. In Appendix A we prove a slightly more general result, encompassing also some multi-dimensional settings, see Proposition A.1 from which one immediately derives Theorem 2.1 by setting $\Omega=(-L, L)$ and $g \equiv 0$.

Note that Theorem 2.1 was used in the previous work [6] in time $T>L^{2} / \pi$ in order to describe the reachable set of the one-dimensional heat equation. Estimate 2.2 is somehow a Carleman estimate even if here no parameter appears in the proof. In fact, it rather is a limiting Carleman estimate as the conjugated operator (2.3) does not satisfy the usual strict pseudo-convexity conditions of Hörmander 23. We refer in particular to [8] for other instances of limiting Carleman weights in another context, namely elliptic operators.

The second step of our analysis amounts to realize that the solutions $z$ of 2.3 could be explicitly solved using Fourier analysis if one extends the solution $z$ of 2.3 by zero outside the space interval $(-L, L)$. We therefore introduce, for $t \in(0, T]$,

$$
w(t, x)= \begin{cases}z(t, x) & \left(=u(t, x) \exp \left(\frac{x^{2}-L^{2}}{4 t}\right)\right) \\ 0 & \text { for } x \in(-L, L), \\ & \text { for } x \notin(-L, L) .\end{cases}
$$

This function $w$ satisfies the following equation:

$$
\begin{cases}\partial_{t} w+\frac{x}{t} \partial_{x} w+\frac{1}{2 t} w-\partial_{x}^{2} w-\frac{L^{2}}{4 t^{2}} w=\partial_{x} u(t, L) \delta_{L}-\partial_{x} u(t,-L) \delta_{-L}, & (t, x) \in(0, \infty) \times \mathbb{R}, \\ w(0, x)=0, & x \in \mathbb{R} .\end{cases}
$$

Using Fourier transform, one can then compute explicitly

$$
\widehat{w}(T, \xi)=\int_{\mathbb{R}} w(T, x) e^{-\imath \xi x} d x,
$$

at least for some frequency $\xi \in \mathbb{C}$ :

Proposition 2.2. For $\alpha \geqslant 0$, define the sets (see Figure 1)

$$
\mathscr{C}_{\alpha}=\left\{\xi=a+\imath b \in \mathbb{C},(a, b) \in \mathbb{R}^{2} \text {, with }|a| \geqslant|b|+\alpha\right\} .
$$

Let $w$ be given by (2.4) corresponding to some smooth solution $u$ of (1.1).

Then, for any $\xi \in \mathscr{C}_{L /(2 T)}$,

$$
\widehat{w}(T, \xi)=\int_{0}^{T} \sqrt{\frac{T}{t}}\left(-\partial_{x} u(t,-L) e^{\imath \frac{\xi L T}{t}}+\partial_{x} u(t, L) e^{-\imath \frac{\xi L T}{t}}\right) e^{-\left(\xi^{2} T^{2}-\frac{L^{2}}{4}\right)\left(\frac{1}{t}-\frac{1}{T}\right)} d t .
$$

In particular, for any $\alpha>L /(2 T)$, setting

$$
C_{\alpha}(T)=\frac{1}{\sqrt{L(\alpha-L /(2 T))}},
$$

for all $\xi \in \mathscr{C}_{\alpha}$, we have

$$
|\widehat{w}(T, \xi)| \leqslant C_{\alpha}(T) \sqrt{T} e^{|\Im(\xi)| L}\left(\left\|\partial_{x} u(\cdot, L)\right\|_{L^{2}(0, T)}+\left\|\partial_{x} u(\cdot,-L)\right\|_{L^{2}(0, T)}\right) .
$$


The proof of Proposition 2.2 is done in Section 3.1 and relies on explicit computations. In particular, it gives a precise $L^{\infty}$ bound on the high-frequency component of $w(T)$ given by 2.4 corresponding to a smooth solution $u$ of 1.1 .

The third step of our analysis consists in the recovery of the low frequency part of $w$ given by (2.4). In order to do that, we recall that $\widehat{w}(T, \cdot)$ is the Fourier transform of a function supported in $[-L, L]$. Therefore, its growth as $|\Im(\xi)| \rightarrow \infty$ is known, while $\widehat{w}(T, \cdot)$ is holomorphic in the whole complex plane $\mathbb{C}$. Combined with the fact that we have nice estimates on $\widehat{w}(T, \cdot)$ in $\mathscr{C}_{\alpha}$ for $\alpha>L^{2} /(2 T)$, we are in position to use Phragmén-Lindelöf principles to estimate $\widehat{w}(T, \cdot)$ everywhere in the complex plane, but more importantly on the real axis $\mathbb{R}$.

Proposition 2.3. Let $L>0, \alpha>0$ and $f$ be an holomorphic function on $\mathscr{O}_{\alpha}=\mathbb{C} \backslash \mathscr{C}_{\alpha}$ (see Figure 1 ) such that:

- There exists a constant $C_{0}$ such that

$$
\forall \xi \in \partial \mathscr{O}_{\alpha}, \quad|f(\xi)| \leqslant C_{0} \exp (|\Im(\xi)| L)
$$

- There exists a constant $C_{1}$ such that

$$
\forall \xi \in \mathscr{O}_{\alpha}, \quad|f(\xi)| \leqslant C_{1} \exp (|\Im(\xi)| L) .
$$

Denoting by

$$
\tilde{\mathscr{O}}_{1}=\left\{(a, b) \in \mathbb{R}^{2}, \text { such that }|a|<|b|+1\right\},
$$

there exists a unique function $\tilde{\varphi}$ satisfying

$$
\begin{cases}\Delta \tilde{\varphi}=-2 \delta_{(-1,1) \times\{0\}} & \text { in } \tilde{\mathscr{O}}_{1}, \\ \tilde{\varphi}=0 & \text { on } \partial \tilde{\mathscr{O}}_{1}, \\ \lim _{|b| \rightarrow \infty} \sup _{a \in(-|b|-1,|b|+1)}|\tilde{\varphi}(a, b)|=0, & \end{cases}
$$

and we define the function $\varphi$ on $\mathscr{O}_{1}$ as follows:

$$
\varphi(\xi)=\tilde{\varphi}(\Re(\xi), \Im(\xi)), \quad \xi \in \mathscr{O}_{1} .
$$

Then we have the following bound:

$$
\forall \xi \in \mathscr{O}_{\alpha}, \quad|f(\xi)| \leqslant C_{0} \exp (|\Im(\xi)| L) \exp \left(L \alpha \varphi\left(\frac{\xi}{\alpha}\right)\right) .
$$

Besides, the maximum of $\varphi$ on $\mathscr{O}_{1}$ is attained in 0 :

$$
\sup _{\mathscr{O}_{1}} \varphi=\varphi(0)=\frac{\Gamma(1 / 4)^{2}}{4 \sqrt{2} \pi^{2}} \sum_{n \in \mathbb{N}} \frac{(-1)^{n}}{(2 n+1)} \frac{\Gamma(n+1 / 4)}{\Gamma(n+7 / 4)}, \quad(\simeq 0.893204),
$$

which can be alternatively written as

$$
\varphi(0)=\frac{2}{\pi} \frac{\int_{0}^{\frac{\pi}{2}} \ln \left(\cot \left(\frac{t}{2}\right)\right) \sqrt{\cos (t)} d t}{\int_{0}^{\frac{\pi}{2}} \sqrt{\cos (t)} d t} .
$$

Proposition 2.3 mainly reduces to the application of Phragmén-Lindelöf principle for holomorphic functions. In fact, the main point in Proposition 2.3 is that the maximum of the harmonic function $\tilde{\varphi}$ can be explicitly computed. This is done using conformal maps to link the solution of the Laplace equation in the domain $\tilde{\mathscr{O}}_{1}$ with solutions of the Laplace operator in the half-strip, in which explicit solutions can be computed using Fourier decomposition techniques. We refer to Section 3.2 for the proof of Proposition 2.3 


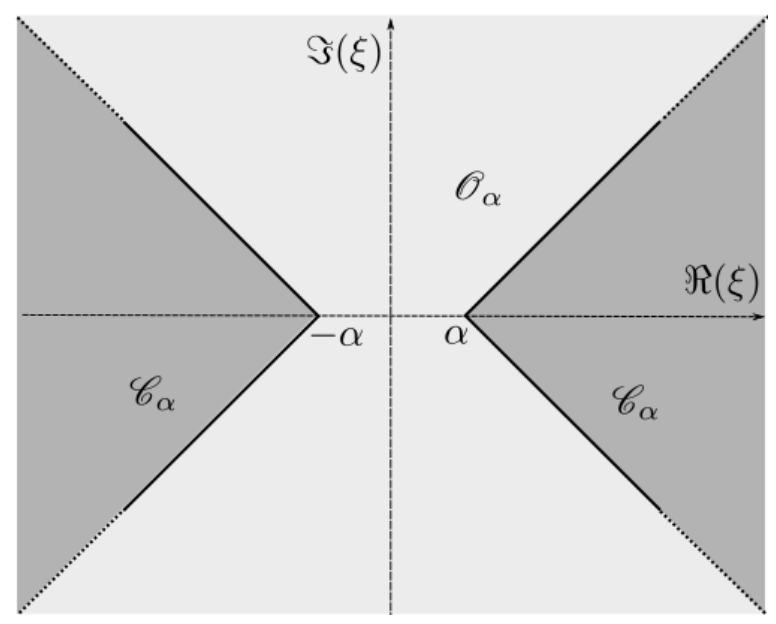

Figure 1: The complex plane, with domains $\mathscr{C}_{\alpha}$ and $\mathscr{O}_{\alpha}$.

Of course, we shall apply Proposition 2.3 to the function $f=\widehat{w}(T, \cdot)$, which, according to $(2.9)$, satisfies 2.10 for any $\alpha>L /(2 T)$ with

$$
C_{0}=C_{\alpha}(T) \sqrt{T}\left(\left\|\partial_{x} u(\cdot, L)\right\|_{L^{2}(0, T)}+\left\|\partial_{x} u(\cdot,-L)\right\|_{L^{2}(0, T)}\right),
$$

while 2.11 holds with

$$
C_{1}=\|w(T)\|_{L^{1}(-L, L)} \leqslant \sqrt{2 L}\|u(T)\|_{L^{2}(-L, L)} \leqslant \sqrt{2 L}\left\|u_{0}\right\|_{L^{2}(-L, L)} .
$$

We then immediately deduce the following corollary.

Corollary 2.4. Let $w$ be given by 2.4 corresponding to some smooth solution $u$ of (1.1). Then, for any $\alpha>L /(2 T)$,

$$
\forall \xi \in \mathscr{O}_{\alpha} \cap \mathbb{R}, \quad|\widehat{w}(T, \xi)| \leqslant C_{\alpha}(T) \sqrt{T} e^{L \alpha \varphi(0)}\left(\left\|\partial_{x} u(\cdot, L)\right\|_{L^{2}(0, T)}+\left\|\partial_{x} u(\cdot,-L)\right\|_{L^{2}(0, T)}\right),
$$

where $C_{\alpha}(T)$ denotes the constant in 2.8 .

End of the proof of Theorem 1.1. Let $\varepsilon>0$, and choose $\alpha=(1+\varepsilon) L /(2 T)$. Combining 2.17) and (2.9), we see that

$$
\forall \xi \in \mathbb{R}, \quad|\widehat{w}(T, \xi)| \leqslant \sqrt{\frac{2}{\varepsilon}} \frac{T}{L} \exp \left((1+\varepsilon) \frac{L^{2}}{2 T} \varphi(0)\right)\left(\left\|\partial_{x} u(\cdot, L)\right\|_{L^{2}(0, T)}+\left\|\partial_{x} u(\cdot,-L)\right\|_{L^{2}(0, T)}\right) .
$$

Then, using Theorem 2.1 and the identity

$$
\int_{-L}^{L}\left|\partial_{x} z(T, x)\right|^{2} d x-\frac{L^{2}}{4 T^{2}} \int_{-L}^{L}|z(T, x)|^{2} d x=\int_{\mathbb{R}}\left(|\xi|^{2}-\frac{L^{2}}{4 T^{2}}\right)|\widehat{w}(T, \xi)|^{2} d \xi
$$

we have

$$
\frac{3 L^{2}}{4 T^{2}} \int_{|\xi|>L / T}|\widehat{w}(T, \xi)|^{2} d \xi \leqslant \frac{L}{T}\left(\left\|\partial_{x} u(\cdot, L)\right\|_{L^{2}(0, T)}^{2}+\left\|\partial_{x} u(\cdot,-L)\right\|_{L^{2}(0, T)}^{2}\right)+\frac{L^{2}}{4 T^{2}} \int_{|\xi|<L /(2 T)}|\widehat{w}(T, \xi)|^{2} d \xi
$$

Combined with 2.18, we obtain

$$
\int_{|\xi|>L / T}|\widehat{w}(T, \xi)|^{2} d \xi \leqslant\left(\frac{4 T}{3 L}+\frac{4 T}{3 L \varepsilon} \exp \left((1+\varepsilon) \frac{L^{2}}{T} \varphi(0)\right)\right)\left(\left\|\partial_{x} u(\cdot, L)\right\|_{L^{2}(0, T)}^{2}+\left\|\partial_{x} u(\cdot,-L)\right\|_{L^{2}(0, T)}^{2}\right),
$$


and

$$
\int_{|\xi|<L / T}|\widehat{w}(T, \xi)|^{2} d \xi \leqslant \frac{8 T}{\varepsilon L} \exp \left((1+\varepsilon) \frac{L^{2}}{T} \varphi(0)\right)\left(\left\|\partial_{x} u(\cdot, L)\right\|_{L^{2}(0, T)}^{2}+\left\|\partial_{x} u(\cdot,-L)\right\|_{L^{2}(0, T)}^{2}\right) .
$$

Using Parseval identity and the explicit form of $w$ in 2.4 , we easily get, for some constant $C_{\varepsilon}(T)$ that goes to zero as $T \rightarrow 0$, that

$$
\begin{aligned}
& \left\|u(T, x) \exp \left(\frac{x^{2}-L^{2}}{4 T}\right)\right\|_{L^{2}(-L, L)} \\
& \qquad \leqslant C_{\varepsilon}(T) \exp \left(\frac{L^{2}}{2 T}(1+\varepsilon) \varphi(0)\right)\left(\left\|\partial_{x} u(\cdot, L)\right\|_{L^{2}(0, T)}+\left\|\partial_{x} u(\cdot,-L)\right\|_{L^{2}(0, T)}\right)
\end{aligned}
$$

which we rewrite as

$$
\begin{aligned}
& \left\|u(T, x) \exp \left(\frac{x^{2}}{4 T}\right)\right\|_{L^{2}(-L, L)} \\
& \quad \leqslant C_{\varepsilon}(T) \exp \left(\frac{L^{2}}{T}\left(\frac{1}{4}+\frac{1}{2}(1+\varepsilon) \varphi(0)\right)\right)\left(\left\|\partial_{x} u(\cdot, L)\right\|_{L^{2}(0, T)}+\left\|\partial_{x} u(\cdot,-L)\right\|_{L^{2}(0, T)}\right) .
\end{aligned}
$$

This concludes the proof of Theorem 1.1, as $C_{\varepsilon}(T) \leqslant C_{\varepsilon}(1)=C_{\varepsilon}$ for $T$ small enough, for some $C_{\varepsilon}$ independent of $T$.

Remark 2.5. Note that the constant $C_{\varepsilon}$ in the above proof blows up as $\varepsilon$ goes to zero. If it were not the case, one could pass to the limit $\varepsilon \rightarrow 0$ in [2.21), so that one could choose $K=K_{0}$ in Theorem 1.1 . So far, we do not know if this choice is allowed in Theorem 1.1 or not.

We have thus reduced the proof of Theorem 1.1 to the proofs of Theorem 2.1 Propositions 2.2 and 2.3 The proof of Theorem 2.1 is postponed to Appendix A in which a slightly more general result is proved (Proposition A.1), while the proofs of Propositions 2.2 and 2.3 are detailed in the section afterwards.

Remark 2.6. The above approach allows in fact to recover an explicit formula to compute $\widehat{w}(T)$ in terms of the observations. Namely, for $\xi \in \mathbb{R}$ with $|\xi| \geqslant L /(2 T)$, formula (2.7) yields

$$
\widehat{w}(T, \xi)=\int_{0}^{T} \sqrt{\frac{T}{t}}\left(-\partial_{x} u(t,-L) e^{\imath \frac{\xi L T}{t}}+\partial_{x} u(t, L) e^{-\imath \frac{\xi L T}{t}}\right) e^{-\left(\xi^{2} T^{2}-\frac{L^{2}}{4}\right)\left(\frac{1}{t}-\frac{1}{T}\right)} d t .
$$

On the other hand, combining the formula 2.7) and Remark 3.2 allowing to get an explicit expression under the assumptions of Proposition 2.3. we get: for all $\alpha_{*}>\alpha>L /(2 T)$, for all $\xi \in \mathbb{R}$ with $|\xi|<L /(2 T)$,

$$
\begin{aligned}
\widehat{w}(T, \xi)=-\int_{0}^{T} & \sqrt{\frac{T}{t}} \partial_{x} u(t,-L) \frac{1}{2 \imath \pi} \int_{\gamma_{\alpha}} \frac{e^{L \alpha_{*}(\phi(\xi / \alpha)-\phi(\zeta / \alpha))}}{\zeta-\xi} e^{\imath \frac{\zeta L T}{t}} e^{-\left(\zeta^{2} T^{2}-\frac{L^{2}}{4}\right)\left(\frac{1}{t}-\frac{1}{T}\right)} d \zeta d t \\
& +\int_{0}^{T} \sqrt{\frac{T}{t}} \partial_{x} u(t, L) \frac{1}{2 \imath \pi} \int_{\gamma_{\alpha}} \frac{e^{L \alpha_{*}(\phi(\xi / \alpha)-\phi(\zeta / \alpha))}}{\zeta-\xi} e^{-\imath \frac{\zeta L T}{t}} e^{-\left(\zeta^{2} T^{2}-\frac{L^{2}}{4}\right)\left(\frac{1}{t}-\frac{1}{T}\right)} d \zeta d t
\end{aligned}
$$

where $\phi$ is an holomorphic function on $\mathscr{O}_{1}$ such that $\Re(\phi(\xi))=\varphi(\xi)+|\Im(\xi)|$ for all $\xi \in \mathscr{O}_{1}$ (see Section 3.2.2 for the existence of such function $\phi)$, and $\gamma_{\alpha}$ is the union of the two connected components of $\partial \mathscr{O}_{\alpha}$ oriented counter-clockwise. But these formula does not seem easy to deal with as the kernels

$$
K_{\mp}(t, \xi)=\frac{1}{2 \imath \pi} \int_{\gamma_{\alpha}} \frac{e^{L \alpha_{*}(\phi(\xi / \alpha)-\phi(\zeta / \alpha))}}{\zeta-\xi} e^{ \pm \imath \frac{\zeta L T}{t}} e^{-\left(\zeta^{2} T^{2}-\frac{L^{2}}{4}\right)\left(\frac{1}{t}-\frac{1}{T}\right)} d \zeta, \quad(t, \xi) \in(0, T) \times\left(-\frac{L}{2 T}, \frac{L}{2 T}\right),
$$

are difficult to estimate directly. 


\section{Proof of Theorem 1.1: intermediate results}

\subsection{Proof of Proposition 2.2}

Let $w$ as in Proposition 2.2. Then $w$ satisfies the equation 2.5. When taking its Fourier transform in the space variable, we easily check that

$$
\widehat{w}(t, \xi)=\int_{\mathbb{R}} w(t, x) e^{-\imath \xi x} d x, \quad(t, \xi) \in[0, T] \times \mathbb{R},
$$

solves the equation

$$
\begin{cases}\partial_{t} \widehat{w}-\frac{\xi}{t} \partial_{\xi} \widehat{w}-\frac{1}{2 t} w+\xi^{2} \widehat{w}-\frac{L^{2}}{4 t^{2}} \widehat{w}=\partial_{x} u(t, L) e^{-\imath \xi L}-\partial_{x} u(t,-L) e^{\imath \xi L}, & (t, \xi) \in(0, \infty) \times \mathbb{R}, \\ \widehat{w}(0, \xi)=0, & \xi \in \mathbb{R} .\end{cases}
$$

We are thus back to the study of a transport equation. For each $\xi_{0} \in \mathbb{R}$, we therefore introduce the characteristics $\xi\left(t, \xi_{0}\right)$ reaching $\xi_{0}$ at time $T$ :

$$
\frac{d \xi}{d t}\left(t, \xi_{0}\right)=-\frac{\xi\left(t, \xi_{0}\right)}{t}, \quad t \in(0, T], \quad \xi\left(T, \xi_{0}\right)=\xi_{0},
$$

which is explicitly given by

$$
\xi\left(t, \xi_{0}\right)=\frac{\xi_{0} T}{t}, \quad t \in(0, T]
$$

We can thus write, for all $t \in(0, T]$,

$$
\frac{d}{d t}\left(\widehat{w}\left(t, \frac{\xi_{0} T}{t}\right)\right)+\left(\frac{1}{t^{2}}\left(\xi_{0}^{2} T^{2}-\frac{L^{2}}{4}\right)-\frac{1}{2 t}\right) \widehat{w}\left(t, \frac{\xi_{0} T}{t}\right)=\partial_{x} u(t, L) e^{-\imath \frac{\xi_{0} L T}{t}}-\partial_{x} u(t,-L) e^{\imath \frac{\xi_{0} L T}{t}} .
$$

This yields the formula

$$
\frac{d}{d t}\left(\widehat{w}\left(t, \frac{\xi_{0} T}{t}\right) t^{-1 / 2} e^{-\left(\xi_{0}^{2} T^{2}-L^{2} / 4\right) / t}\right)=\left(\partial_{x} u(t, L) e^{-\imath \frac{\xi_{0} L T}{t}}-\partial_{x} u(t,-L) e^{{ }^{2} \frac{\xi_{0} L T}{t}}\right) t^{-1 / 2} e^{-\left(\xi_{0}^{2} T^{2}-L^{2} / 4\right) / t} .
$$

For any $\eta>0$, we can integrate this formula between $\eta$ and $T$ to get

$$
\begin{aligned}
\widehat{w}\left(T, \xi_{0}\right) T^{1 / 2} e^{-\left(\xi_{0}^{2} T^{2}-L^{2} / 4\right) / T} & -\widehat{w}\left(\eta, \xi_{0}\right) \eta^{1 / 2} e^{-\left(\xi_{0}^{2} T^{2}-L^{2} / 4\right) / \eta} \\
& =\int_{\eta}^{T} t^{-1 / 2}\left(\partial_{x} u(t, L) e^{-\imath \frac{\xi_{0} L T}{t}}-\partial_{x} u(t,-L) e^{{ }^{\frac{\xi_{0} L T}{t}}}\right) e^{-\left(\xi_{0}^{2} T^{2}-L^{2} / 4\right) / t} d t
\end{aligned}
$$

It is not difficult to check that for $\xi_{0} \in \mathbb{R}$ with $\left|\xi_{0}\right|>L /(2 T)$, the integral on the right-hand-side converges when $\eta$ goes to zero, and

$$
\lim _{\eta \rightarrow 0} \widehat{w}\left(\eta, \xi_{0}\right) \eta^{-1 / 2} e^{-\left(\xi_{0}^{2} T^{2}-L^{2} / 4\right) / \eta}=0 .
$$

Therefore, provided $\xi_{0} \in \mathbb{R}$ satisfies $\left|\xi_{0}\right|>L /(2 T)$, one gets the formula

$$
\widehat{w}\left(T, \xi_{0}\right)=\int_{0}^{T} \sqrt{\frac{T}{t}}\left(\partial_{x} u(t, L) e^{-\imath \frac{L \xi_{0} T}{t}}-\partial_{x} u(t,-L) e^{\imath \frac{L \xi_{0} T}{t}}\right) e^{-\left(\xi_{0}^{2} T^{2}-L^{2} / 4\right)(1 / t-1 / T)} d t
$$

This formula coincides with the one in 2.7) for $\xi_{0} \in \mathscr{C}_{L^{+} / 2 T} \cap \mathbb{R}$ (here, we use the notation $L^{+}$to denote any constant strictly larger than $L)$. As $\widehat{w}(T, \cdot)$ is holomorphic on $\mathbb{C}$, we only have to check that the right hand side of formula 3.3 can be extended holomorphically to $\mathscr{C}_{L^{+} / 2 T}$. In fact, writing $\xi=a+\imath b$ with $(a, b) \in \mathbb{R}^{2}$, the right hand side of $(3.3)$ can be extended holomorphically in the domain in which

$$
\left\{\begin{array}{l}
\Re\left(+\imath \xi L T-\left(\xi^{2} T^{2}-\frac{L^{2}}{4}\right)\right)=-b L T-\left(\left(a^{2}-b^{2}\right) T^{2}-\frac{L^{2}}{4}\right)<0, \\
\text { and } \\
\Re\left(-\imath \xi L T-\left(\xi^{2} T^{2}-\frac{L^{2}}{4}\right)\right)=+b L T-\left(\left(a^{2}-b^{2}\right) T^{2}-\frac{L^{2}}{4}\right)<0,
\end{array}\right.
$$


which is equivalent to

$$
|a|>|b|+\frac{L}{2 T},
$$

i.e. $\xi \in \mathscr{C}_{L^{+} /(2 T)}$. We have thus proved that for all $\xi \in \mathscr{C}_{L^{+} /(2 T)}, \widehat{w}(T, \xi)$ is given by the formula 2.7). In fact, by continuity, this formula also holds for $\xi \in \mathscr{C}_{L / 2 T}$.

In order to deduce 2.9 , we start from the formula 2.7 and we use a Cauchy-Schwarz estimate: for $\xi \in \mathscr{C}_{\alpha}$ with $\alpha>L /(2 T)$,

$$
\begin{aligned}
|\widehat{w}(T, \xi)| \leqslant \sqrt{T} & \left\|\partial_{x} u(t, L)\right\|_{L^{2}(0, T)}\left\|t^{-1 / 2} \exp \left(-\frac{i \xi L T}{t}-\left(\xi^{2} T^{2}-\frac{L^{2}}{4}\right)\left(\frac{1}{t}-\frac{1}{T}\right)\right)\right\|_{L^{2}(0, T)} \\
& +\sqrt{T}\left\|\partial_{x} u(t,-L)\right\|_{L^{2}(0, T)}\left\|t^{-1 / 2} \exp \left(+\frac{i \xi L T}{t}-\left(\xi^{2} T^{2}-\frac{L^{2}}{4}\right)\left(\frac{1}{t}-\frac{1}{T}\right)\right)\right\|_{L^{2}(0, T)}
\end{aligned}
$$

Writing $\xi \in \mathscr{C}_{\alpha}$ for $\alpha>L /(2 T)$ as $\xi=a+\imath b$ with $(a, b) \in \mathbb{R}^{2}$ and using the fact that

$$
\begin{aligned}
\Re\left(\mp \imath \xi L T-\left(\xi^{2} T^{2}-\frac{L^{2}}{4}\right)\right) & \leqslant|b| L T-\left(\left(a^{2}-b^{2}\right) T^{2}-\frac{L^{2}}{4}\right) \\
& \leqslant-T^{2}\left(a^{2}-\left(|b|+\frac{L}{2 T}\right)^{2}\right) \\
& \leqslant-T^{2}\left(|a|-\left(|b|+\frac{L}{2 T}\right)\right)\left(|a|+|b|+\frac{L}{2 T}\right) \\
& \leqslant-\frac{L T}{2}\left(\alpha-\frac{L}{2 T}\right),
\end{aligned}
$$

we have the estimates, for $s \in\{-1,1\}$ :

$$
\begin{aligned}
& \left\|t^{-1 / 2} \exp \left(s \frac{\imath \xi L T}{t}-\left(\xi^{2} T^{2}-\frac{L^{2}}{4}\right)\left(\frac{1}{t}-\frac{1}{T}\right)\right)\right\|_{L^{2}(0, T)} \\
& \leqslant\left\|t^{-1 / 2} \exp \left(|b| L+\left(|b| L T-\left(\left(a^{2}-b^{2}\right) T^{2}-\frac{L^{2}}{4}\right)\left(\frac{1}{t}-\frac{1}{T}\right)\right)\right)\right\|_{L^{2}(0, T)} \\
& \leqslant e^{|b| L}\left\|t^{-1 / 2} \exp \left(-\frac{L T}{2}\left(\alpha-\frac{L}{2 T}\right)\left(\frac{1}{t}-\frac{1}{T}\right)\right)\right\|_{L^{2}(0, T)} .
\end{aligned}
$$

Now, doing the change of variable $\mu=L T\left(\alpha-\frac{L}{2 T}\right)\left(\frac{1}{t}-\frac{1}{T}\right)$, we easily get, for all $\xi \in \mathscr{C}_{\alpha}$,

$$
\begin{aligned}
\left\|t^{-1 / 2} \exp \left(-\frac{L T}{2}\left(\alpha-\frac{L}{2 T}\right)\left(\frac{1}{t}-\frac{1}{T}\right)\right)\right\|_{L^{2}(0, T)}^{2} & =\int_{0}^{\infty} e^{-\mu} \frac{d \mu}{\mu+L(\alpha-L /(2 T))} \\
& \leqslant \frac{1}{L(\alpha-L /(2 T))} .
\end{aligned}
$$

Combining (3.4) and this last estimate, we easily conclude estimate 2.9.

\subsection{Proof of Proposition 2.3}

We shall start the proof of Proposition 2.3 by proving the existence of a function $\tilde{\varphi}$ satisfying $(2.12$, and we will then explain how it can be used to derive the bound in 2.14 .

Notations. In the following arguments, to avoid ambiguities, we will write differently complex sets and their identification as a part of $\mathbb{R}^{2}$, for instance denoting $\mathscr{O}_{1}=\{\xi \in \mathbb{C}$, with $|\Re(\xi)|<|\Im(\xi)|+1\}$ and $\tilde{\mathscr{O}}_{1}=\left\{(a, b) \in \mathbb{R}^{2}\right.$, with $\left.|a|<|b|+1\right\}$ as in Proposition 2.3 To be consistent with this notation, we will also distinguish functions of the complex variable $\xi$ from the corresponding ones considered as functions of the real variables $(a, b)$ using a tilde notation for the function viewed as depending on real variables, as in (2.13). 


\subsubsection{Existence and uniqueness of a function $\tilde{\varphi}$ satisfying (2.12)}

The first remark is that the uniqueness of a function $\tilde{\varphi}$ satisfying 2.12 is rather easy to prove. Indeed, if two functions $\tilde{\varphi}_{1}$ and $\tilde{\varphi}_{2}$ satisfy $(2.12)$, then their difference $\tilde{\varphi}_{2}-\tilde{\varphi}_{1}$ is harmonic in $\mathscr{O}_{1}$ and vanishes on $\partial \tilde{\mathscr{O}}_{1}$ as well as at infinity. Therefore, the minimum and maximum of $\tilde{\varphi}_{2}-\tilde{\varphi}_{1}$ is zero, and $\tilde{\varphi}_{1}$ and $\tilde{\varphi}_{2}$ coincide. Thus, we will focus on the existence of a function $\tilde{\varphi}$ as in 2.12 . In fact, by uniqueness, we see that necessarily $\tilde{\varphi}(a, b)=\tilde{\varphi}(a,|b|)$ for all $(a, b) \in \mathscr{O}_{1}$. We will thus only look for a solution $\tilde{\varphi}$ in $\tilde{\mathscr{O}}_{1}^{+}=\tilde{\mathscr{O}}_{1} \cap\left(\mathbb{R} \times \mathbb{R}_{+}^{*}\right)$ of the problem

with the condition at infinity:

$$
\begin{cases}\Delta \tilde{\varphi}=0 & \text { in } \tilde{\mathscr{O}}_{1}^{+} \\ \tilde{\varphi}=0 & \text { on } \partial \tilde{\mathscr{O}}_{1}^{+} \backslash(-1,1) \\ \partial_{b} \tilde{\varphi}(a, 0)=-1 & \text { for } a \in(-1,1)\end{cases}
$$

$$
\lim _{b \rightarrow \infty} \sup _{a \in(-|b|-1,|b|+1)}|\tilde{\varphi}(a, b)|=0
$$

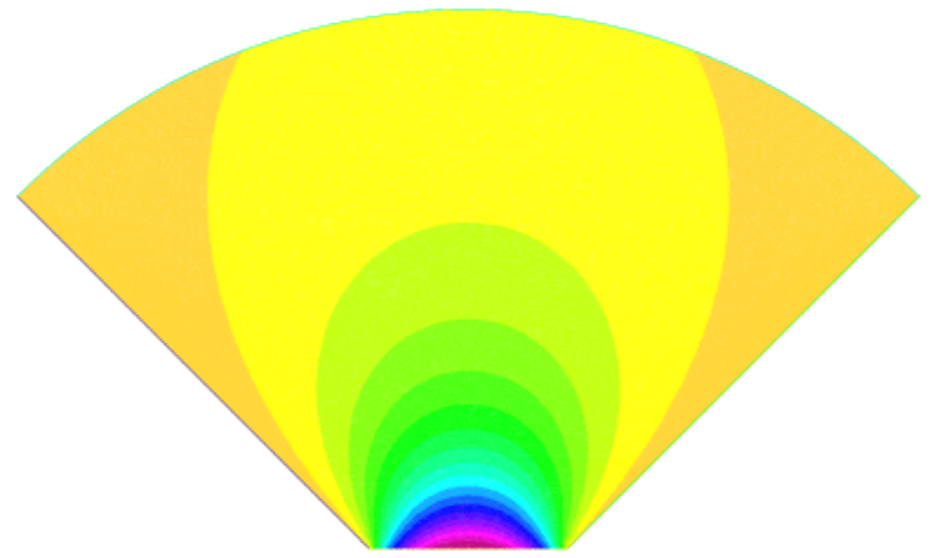

Figure 2: Approximation of $\tilde{\varphi}$ solving [3.5, obtained by a finite element approach (using FreeFem,$++[20$ ).

Let us introduce

$$
\begin{aligned}
& \Gamma_{\ell}=\{\xi \in \mathbb{C}, \text { with } \Im(\xi)>0 \text { and }-\Re(\xi)=1+\Im(\xi)\} \\
& \Gamma_{r}:=\{\xi \in \mathbb{C}, \text { with } \Im(\xi)>0 \text { and } \Re(\xi)=1+\Im(\xi)\}, \\
& \Gamma_{b}:=\{\xi \in \mathbb{C}, \text { with }(\Re(\xi), \Im(\xi)) \in[-1,1] \times\{0\}\},
\end{aligned}
$$

the three components of the boundary of $\mathscr{O}_{1}^{+}=\mathscr{O}_{1} \cap\{\Im(\xi)>0\}$.

Our goal is to show the existence of a function $\tilde{\varphi}$ satisfying 3.5 . In order to do so, we will rely on two Schwarz-Christoffel conformal mappings [21, Chapter 5.12].

The first one, $F_{3 / 4}$, is defined for all $\zeta \in \mathbb{C}^{+}=\{\zeta \in \mathbb{C}, \Im(\zeta) \geqslant 0\}$ by

$$
F_{3 / 4}(\zeta)=\frac{2}{K_{3 / 4}} \int_{-1}^{\zeta}\left(1-z^{2}\right)^{-1 / 4} d z-1, \text { with } K_{3 / 4}=\int_{-1}^{1}\left(1-x^{2}\right)^{-1 / 4} d x=\sqrt{\pi} \frac{\Gamma(3 / 4)}{\Gamma(5 / 4)}
$$

where the path integration is arbitrary in $\mathbb{C}^{+}$.

The map $F_{3 / 4}$ conformally maps $\mathbb{C}^{+}$into $\overline{\mathscr{O}_{1}^{+}}$, and verifies the following properties:

$$
F_{3 / 4}(-1)=-1, \quad F_{3 / 4}(0)=0, \quad F_{3 / 4}(1)=1,
$$

and

$$
F_{3 / 4}((-\infty,-1))=\Gamma_{\ell}, \quad F_{3 / 4}((-1,1))=\Gamma_{b}, \quad F_{3 / 4}((1, \infty))=\Gamma_{r}, \quad F_{3 / 4}\left(\imath \mathbb{R}^{+}\right)=\imath \mathbb{R}^{+}
$$


The second conformal mapping we will use is defined, for any $\zeta \in \mathbb{C}^{+}$, by

$$
F_{1 / 2}(\zeta)=\frac{2}{\pi} \arcsin (\zeta)=\frac{2}{\pi} \int_{-1}^{\zeta}\left(1-z^{2}\right)^{-1 / 2} d z-1
$$

which conformally maps $\mathbb{C}^{+}$into the closure of the half strip $\mathscr{S}_{1}^{+}=\{\Xi=A+\imath B, A \in(-1,1), B>0\}$ with the following properties:

$$
F_{1 / 2}(-1)=-1, \quad F_{1 / 2}(0)=0, \quad F_{1 / 2}(1)=1,
$$

and

$$
\begin{array}{cl}
F_{1 / 2}((-\infty,-1])=-1+\imath \mathbb{R}^{+}, & F_{1 / 2}((-1,1))=(-1,1), \\
F_{1 / 2}([1, \infty))=1+\imath \mathbb{R}^{+}, & F_{1 / 2}\left(\imath \mathbb{R}^{+}\right)=\imath \mathbb{R}^{+} .
\end{array}
$$

Finally, we define the conformal mapping

$$
F=F_{1 / 2} \circ F_{3 / 4}^{-1}
$$

which maps $\mathscr{O}_{1}^{+}$into $\mathscr{S}_{1}^{+}$.

For any $\xi=a+\imath b \in \mathscr{O}_{1}^{+}$, we denote $\Xi=A+\imath B=F(\xi)$. Using standard computation from conformal transplantation [21. Chapter 5.6], we see that $\tilde{\varphi}$ solves 3.5 in $\tilde{\mathscr{O}}_{1}^{+}$if and only if $\tilde{\Phi}$ given by $\tilde{\Phi}(A, B)=\tilde{\varphi}(a, b)$ for $A+\imath B=F(a+\imath b)$ solves the following problem posed in the half-strip $\tilde{\mathscr{S}}_{1}^{+}$:

$$
\begin{cases}\Delta_{A, B} \tilde{\Phi}=0, & \text { for } A \in(-1,1), B>0, \\ \tilde{\Phi}(-1, B)=\tilde{\Phi}(1, B)=0, & \text { for } B>0, \\ \partial_{B} \tilde{\Phi}(A, 0)=-\frac{\pi}{K_{3 / 4}} \sqrt{\cos \left(\frac{\pi}{2} A\right),} & \text { for } A \in(-1,1) .\end{cases}
$$

If the first two equations are standard, the last one deserves additional details. In fact, it comes from the identity [21, Theorem 5.6a]

$$
\operatorname{grd}_{\xi} \varphi(\xi)=\operatorname{grd}_{\Xi} \Phi(F(\xi)) \overline{F^{\prime}(\xi)}
$$

applied to $\xi=a \in(-1,1)$, (implying $F(\xi)=A \in(-1,1)$ ), where grd is the complex gradient: for $\xi=a+\imath b$, $\operatorname{grd}_{\xi} \varphi(\xi)=\partial_{a} \tilde{\varphi}(a, b)+\imath \partial_{b} \tilde{\varphi}(a, b)$ and for $\Xi=A+\imath B, \operatorname{grd}_{\Xi} \Phi(\Xi)=\partial_{A} \tilde{\Phi}(A, B)+\imath \partial_{B} \tilde{\Phi}(A, B)$.

We therefore have to compute $F^{\prime}(\xi)=\left(F_{1 / 2} \circ F_{3 / 4}^{-1}\right)^{\prime}(\xi)=F_{1 / 2}^{\prime}\left(F_{3 / 4}^{-1}(\xi)\right)\left(F_{3 / 4}^{-1}\right)^{\prime}(\xi)$. To do so, let us define $\zeta=F_{3 / 4}^{-1}(\xi) \in \mathbb{C}^{+}$. By definition,

$$
F_{1 / 2}^{\prime}\left(F_{3 / 4}^{-1}(\xi)\right)=F_{1 / 2}^{\prime}(\zeta)=\frac{2}{\pi} \frac{1}{\sqrt{1-\zeta^{2}}}
$$

whereas

$$
\left(F_{3 / 4}^{-1}\right)^{\prime}(\xi)=\left(F_{3 / 4}^{-1}\right)^{\prime}\left(F_{3 / 4}(\zeta)\right)=\frac{1}{F_{3 / 4}^{\prime}(\zeta)}=\frac{K_{3 / 4}}{2} \sqrt[4]{1-\zeta^{2}}
$$

Therefore,

$$
F^{\prime}(\xi)=\frac{K_{3 / 4}}{\pi} \frac{1}{\sqrt[4]{1-\zeta^{2}}}
$$

with $\zeta=F_{3 / 4}^{-1}(\xi)$. In particular, for $\xi=a \in(-1,1), \zeta \in(-1,1)$ and therefore $F^{\prime}(\xi) \in \mathbb{R}$ and

$$
\partial_{B} \tilde{\Phi}(A, 0)=\partial_{b} \tilde{\varphi}(a, 0) \frac{1}{F^{\prime}(a)}=-\frac{\pi}{K_{3 / 4}} \sqrt[4]{1-\zeta^{2}}, \text { with } \zeta=F_{3 / 4}^{-1}(a)
$$

To conclude, we just note that $\zeta=F_{1 / 2}^{-1}(A)$ if and only if $\zeta=\sin (A \pi / 2)$, and the identity 3.7 (3) follows. Problem (3.7) has the advantage of being explicitly solvable. Indeed, as $\tilde{\Phi}$ is harmonic in $(-1,1) \times(0, \infty)$, and verifies $\Phi(-1, B)=\tilde{\Phi}(1, B)=0$ for all $B>0$, it necessarily has the following decomposition:

$$
\tilde{\Phi}(A, B)=\sum_{k \geqslant 1}\left(\alpha_{k} e^{-k \frac{\pi}{2} B}+a_{k} e^{k \frac{\pi}{2} B}\right) \sin \left(k \frac{\pi}{2}(A+1)\right), \quad(A, B) \in \tilde{\mathscr{S}}_{1}^{+} .
$$


Recalling (3.6) on $\tilde{\varphi}$, we wish to have $\tilde{\Phi}$ going to zero as $B \rightarrow \infty$. We thus choose $a_{k}=0$ for all $k \geqslant 1$, so that $\tilde{\Phi}$ writes:

$$
\tilde{\Phi}(A, B)=\sum_{k \geqslant 1} \alpha_{k} e^{-k \frac{\pi}{2} B} \sin \left(k \frac{\pi}{2}(A+1)\right), \quad(A, B) \in \tilde{\mathscr{S}}_{1}^{+}
$$

But the boundary condition on $B=0$ is equivalent to

$$
\frac{\pi}{2} \sum_{k \geqslant 1} k \alpha_{k} \sin \left(k \frac{\pi}{2}(A+1)\right)=\frac{\pi}{K_{3 / 4}} \sqrt{\cos \left(\frac{\pi}{2} A\right)},
$$

which explicitly yields the coefficients $\alpha_{k}$ :

$$
\forall k \in \mathbb{N}, \quad \alpha_{k}=\frac{2}{k} \frac{1}{K_{3 / 4}} \int_{-1}^{1} \sin \left(k \frac{\pi}{2}(A+1)\right) \sqrt{\cos \left(\frac{\pi}{2} A\right)} d A .
$$

As $\sqrt{\cos (A \pi / 2)}$ is an even function and $\sin (k \pi(A+1) / 2)$ is an odd function for all even $k$, we have $\alpha_{k}=0$ for all even $k$. On the other hand, we have for any $n \in \mathbb{N}$ (see [18, equation 3.631.9]),

$$
\begin{aligned}
\int_{-1}^{1} \sin \left((2 n+1) \frac{\pi}{2}(A+1)\right) \sqrt{\cos \left(\frac{\pi}{2} A\right)} d A & =(-1)^{n} \int_{-1}^{1} \cos \left((2 n+1) \frac{\pi}{2} A\right) \sqrt{\cos \left(\frac{\pi}{2} A\right)} d A \\
& =(-1)^{n} \frac{4}{\pi} \int_{0}^{\frac{\pi}{2}} \cos ((2 n+1) t) \sqrt{\cos (t)} d t \\
& =\frac{1}{2 \sqrt{\pi}} \frac{\Gamma\left(n+\frac{1}{4}\right)}{\Gamma\left(n+\frac{7}{4}\right)}
\end{aligned}
$$

where $\Gamma(\cdot)$ stands for the Gamma function, so in the end we obtain

$$
\alpha_{2 n+1}=\frac{1}{\pi} \frac{1}{2 n+1} \frac{\Gamma\left(\frac{5}{4}\right)}{\Gamma\left(\frac{3}{4}\right)} \frac{\Gamma\left(n+\frac{1}{4}\right)}{\Gamma\left(n+\frac{7}{4}\right)},
$$

which can be slightly simplified using that $\Gamma(5 / 4)=\Gamma(1 / 4) / 4$ and $\Gamma(3 / 4)=\sqrt{2} \pi / \Gamma(1 / 4)$, giving

$$
\alpha_{2 n+1}=\frac{\Gamma\left(\frac{1}{4}\right)^{2}}{4 \sqrt{2} \pi^{2}} \frac{1}{(2 n+1)} \frac{\Gamma\left(n+\frac{1}{4}\right)}{\Gamma\left(n+\frac{7}{4}\right)} .
$$

So finally, we have

$$
\tilde{\Phi}(A, B)=\frac{\Gamma\left(\frac{1}{4}\right)^{2}}{4 \sqrt{2} \pi^{2}} \sum_{n \in \mathbb{N}} \frac{1}{(2 n+1)} \frac{\Gamma\left(n+\frac{1}{4}\right)}{\Gamma\left(n+\frac{7}{4}\right)} e^{-(2 n+1) \frac{\pi}{2} B} \sin \left((2 n+1) \frac{\pi}{2}(A+1)\right), \quad(A, B) \in \mathscr{S}_{1}^{+},
$$

and

$$
\tilde{\Phi}(0,0)=\frac{\Gamma\left(\frac{1}{4}\right)^{2}}{4 \sqrt{2} \pi^{2}} \sum_{n \in \mathbb{N}} \frac{(-1)^{n}}{(2 n+1)} \frac{\Gamma\left(n+\frac{1}{4}\right)}{\Gamma\left(n+\frac{7}{4}\right)} .
$$

Note that, according to [26, 1.4.25],

$$
\frac{1}{2 n+1} \frac{\Gamma\left(n+\frac{1}{4}\right)}{\Gamma\left(n+\frac{7}{4}\right)} \underset{n \rightarrow \infty}{\simeq} \frac{1}{2 n^{\frac{5}{2}}}
$$

hence the above series are well defined. In particular, the identity 3.9 can be understood pointwise and $\tilde{\Phi}(\cdot, B)$ goes to zero as $B \rightarrow \infty$ :

$$
\sup _{A \in(-1,1)}\left\{|\tilde{\Phi}(A, B)|+\left|\partial_{A} \tilde{\Phi}(A, B)\right|\right\} \leqslant C \exp (-\pi B / 2), \quad B \geqslant 0 .
$$

Let us also note that, because $\tilde{\Phi}(0,0)$ is defined through a converging alternating series, we have

$$
\tilde{\Phi}(0,0)<\frac{\Gamma\left(\frac{1}{4}\right)^{2}}{4 \sqrt{2} \pi^{2}} \sum_{n=0}^{2} \frac{(-1)^{n}}{(2 n+1)} \frac{\Gamma\left(n+\frac{1}{4}\right)}{\Gamma\left(n+\frac{7}{4}\right)}<\frac{9}{10} .
$$


Computing the 100th partial sum of the series using Octave 9], we obtain

$$
\tilde{\Phi}(0,0) \sim 0.893204
$$

A different expression for $\tilde{\Phi}(0,0)$ is the following:

$$
\tilde{\Phi}(0,0)=\frac{2}{\pi} \frac{\int_{0}^{\frac{\pi}{2}} \ln \left(\cot \left(\frac{t}{2}\right)\right) \sqrt{\cos (t)} d t}{\int_{0}^{\frac{\pi}{2}} \sqrt{\cos (t)} d t},
$$

which easily comes from the equality $\tilde{\Phi}(0,0)=\sum_{n \in \mathbb{N}}(-1)^{n} \alpha_{2 n+1}$, the fact that

$$
\alpha_{2 n+1}=(-1)^{n} \frac{8}{(2 n+1) \pi} \frac{1}{K_{3 / 4}} \int_{0}^{\frac{\pi}{2}} \cos ((2 n+1) t) \sqrt{\cos (t)} d t
$$

the definition of $K_{3 / 4}$ and the identity (see [18, identity 1.442 .2 p. 46])

$$
\sum_{n \in \mathbb{N}} \frac{\cos ((2 n+1) t)}{2 n+1}=\frac{1}{2} \ln \left(\cot \left(\frac{t}{2}\right)\right) .
$$

Note in particular that under the form 3.12 , one immediately checks that

$$
\tilde{\Phi}(0,0)>0
$$

In agreement with Figure 2, we then show that the maximum of $\tilde{\Phi}$ is attained at $(A, B)=(0,0)$. We first note that the function $\tilde{\Phi}$ given by $\sqrt{3.9}$ is positive in the strip $\tilde{\mathscr{S}}_{1}^{+}$. Indeed, since $\tilde{\Phi}$ is harmonic in the half strip $\tilde{\mathscr{S}}_{1}^{+}$and is not constant, its minimum is attained at the boundary $\tilde{\mathscr{S}}_{1}^{+}$or at infinity 15 , Lemma 3.4 $\&$ Theorem 3.5]. The boundary conditions on $\partial \tilde{\mathscr{S}}_{1}^{+}$and the behavior of $\tilde{\Phi}$ as $B \rightarrow \infty$ in (3.11) implies that the minimum value of $\tilde{\Phi}$ is 0 and is attained on the lateral boundaries $\{-1,1\} \times \mathbb{R}_{+}$of the half strip. Consequently, the function $\tilde{\Phi}$ is positive in $\tilde{\mathscr{S}}_{1}^{+}$, and its minimal value is 0 . Besides, as $\tilde{\Phi}$ vanishes on the lateral boundaries $\{-1,1\} \times \mathbb{R}^{+}$of the half strip, $\partial_{A} \tilde{\Phi}(1, \cdot)$ is strictly negative by Hopf maximum principle 35. Chapter 2, Theorem 7]. We then consider the function $\tilde{\Phi}_{A}=\partial_{A} \tilde{\Phi}$. Formula 3.9 easily yields that $\tilde{\Phi}_{A}(0, B)=0$ for $B>0$, so that $\tilde{\Phi}_{A}$ satisfies:

$$
\begin{cases}\Delta \tilde{\Phi}_{A}=0 & \text { in } \tilde{\mathscr{S}}_{1}^{+} \cap\{A>0\} \\ \tilde{\Phi}_{A}(0, B)=0 & \text { for } B>0 \\ \tilde{\Phi}_{A}(1, B)<0 & \text { for } B>0 \\ \partial_{B} \tilde{\Phi}_{A}(A, 0) \geqslant 0 & \text { for } A \in(0,1), \\ \lim _{|B| \rightarrow \infty} \sup _{A \in(0,1)}\left|\tilde{\Phi}_{A}(A, B)\right|=0 . & \end{cases}
$$

It easily follows that the maximum of $\tilde{\Phi}_{A}$ is necessarily non-positive in $\tilde{\mathscr{S}}_{1}^{+} \cap\{A>0\}$ by the application of the maximum principle. As $\tilde{\Phi}$ is harmonic in the half-strip $\tilde{\mathscr{S}}_{1}^{+}$and is strictly positive in $(0,0)$ (see equation (3.13), the maximum of $\tilde{\Phi}$ on the half strip $\tilde{\mathscr{S}}_{1}^{+}$is necessarily attained on the boundary of the half-strip or at infinity, therefore on $(-1,1) \times\{0\}$ according to the boundary conditions satisfied by $\tilde{\Phi}$ in 3.7 and the conditions (3.11) as $B \rightarrow \infty$. Now, $\partial_{A} \tilde{\Phi}$ is non-positive in $\tilde{\mathscr{S}}_{1}^{+} \cap\{A>0\}$ and $\tilde{\Phi}(A, B)=\tilde{\Phi}(|A|, B)$ in the half-strip $\mathscr{S}_{1}^{+}$according to 3.9$)$, so the maximum of $\tilde{\Phi}$ is necessarily attained in $(A, B)=(0,0){ }^{1}$

We then come back to the problem $3.5-3.6$ and check that the function $\tilde{\varphi}$ given by

$$
\tilde{\varphi}(a, b)=\tilde{\Phi}(A, B), \text { for } A+\imath B=F(a+\imath b), \quad(a, b) \in \tilde{\mathscr{O}}_{1}^{+},
$$

with $\tilde{\Phi}$ as in 3.9 , satisfies $3.5-3.6$.

By construction, $\tilde{\varphi}$ automatically satisfies 3.5 and its maximum is attained in $(a, b)=(0,0)$ and takes

\footnotetext{
${ }^{1}$ We are indebted to Jean-Michel Roquejoffre for this elegant proof of the fact that the maximum of $\tilde{\Phi}$ is attained in $(0,0)$.
} 
value $\tilde{\varphi}(0,0)=\tilde{\Phi}(0,0)$. We thus only have to check the condition $(3.6)$. In order to do that, let us introduce the real functions $\tilde{A}=\tilde{A}(a, b)$ and $\tilde{B}=\tilde{B}(a, b)$ given for $(a, b) \in \tilde{\mathscr{O}}_{1}^{+}$by

$$
F(a+\imath b)=\tilde{A}(a, b)+\imath \tilde{B}(a, b)
$$

and let us check that

$$
\lim _{b \rightarrow \infty} \inf _{|a|<b+1} \tilde{B}(a, b)=+\infty .
$$

Indeed, if it were not the case, we could find real sequences $\left(a_{n}, b_{n}\right)_{n \in \mathbb{N}}$ with

$$
\lim _{n \rightarrow \infty} b_{n}=+\infty, \quad \forall n \in \mathbb{N}, \quad\left|a_{n}\right| \leqslant b_{n}+1 \quad \text { and } \quad \sup _{n} \tilde{B}\left(a_{n}, b_{n}\right)<\infty .
$$

Then, if we set $\zeta_{n}=F_{3 / 4}^{-1}\left(a_{n}+\imath b_{n}\right)$, by construction,

$$
F_{1 / 2}\left(\zeta_{n}\right)=\tilde{A}\left(a_{n}, b_{n}\right)+\imath \tilde{B}\left(a_{n}, b_{n}\right)
$$

Therefore, according to the definition of $F_{1 / 2}$,

$$
\zeta_{n}=\sin \left(\frac{\pi}{2}\left(\tilde{A}\left(a_{n}, b_{n}\right)+\imath \tilde{B}\left(a_{n}, b_{n}\right)\right)\right),
$$

so that the sequence $\left(\zeta_{n}\right)$ is uniformly bounded in $\mathbb{C}$ as $n \rightarrow \infty$. Then the sequence $\left(a_{n}, b_{n}\right)$ is given by $a_{n}+\imath b_{n}=F_{3 / 4}\left(\zeta_{n}\right)$. But $F_{3 / 4}$ maps bounded sets of $\mathbb{C}$ into bounded sets of $\mathbb{C}$, so this is in contradiction with (3.17), and the property (3.16) holds.

We can thus use 3.11 to get that for all $b \geqslant 0$,

$$
\sup _{|a|<b+1}\{|\tilde{\varphi}(a, b)|\} \leqslant C \exp \left(-\frac{\pi}{2} \inf _{|a|<b+1} \tilde{B}(a, b)\right),
$$

which, according to 3.16, implies 3.6.

Remark 3.1. Another approach to obtain informations on $\tilde{\varphi}$ solution of 3.5 is through integral equations. More precisely, let us define, for $\left((a, b),\left(a_{0}, b_{0}\right)\right) \in\left(\tilde{\mathscr{O}}_{1}^{+}\right)^{2}$, we define $\mathcal{G}$ as follows:

$$
\tilde{\mathcal{G}}\left(a, b, a_{0}, b_{0}\right)=\frac{1}{4 \pi} \ln \left(\frac{\left(\left(a-a_{0}\right)^{2}+\left(b-b_{0}\right)^{2}\right)\left(\left(a+a_{0}\right)^{2}+\left(b+b_{0}+2\right)^{2}\right)}{\left(\left(a+b_{0}+1\right)^{2}+\left(b+a_{0}+1\right)^{2}\right)\left(\left(a-b_{0}-1\right)^{2}+\left(a_{0}-b-1\right)^{2}\right)}\right) .
$$

It is readily verified that for any $\left(a_{0}, b_{0}\right) \in \tilde{\mathscr{O}}_{1}^{+}, \tilde{\mathcal{G}}\left(\cdot, \cdot, a_{0}, b_{0}\right)$ verifies

$$
\begin{cases}\Delta_{a, b} \tilde{\mathcal{G}}\left(\cdot, \cdot, a_{0}, b_{0}\right)=\delta_{\left(a_{0}, b_{0}\right)} & \text { in } \tilde{\mathscr{O}}_{1}^{+} \\ \tilde{\mathcal{G}}\left(a, b, a_{0}, b_{0}\right)=0 & \text { for }(a, b) \text { such that }|a|=|b|+1 .\end{cases}
$$

Indeed, this comes from the fact that $\tilde{\mathcal{G}}$ is the suitable combination of the fundamental solution of the Laplace operator in the sectors $\left\{(a, b) \in \mathbb{R}^{2}\right.$, with $\left.b=|a|-1\right\}$ and $\left\{(a, b) \in \mathbb{R}^{2}\right.$, with $\left.b=1-|a|\right\}$.

Then, standard computations show that $\tilde{\varphi}$ is a solution of 3.5 if and only if it verifies the integral equation

$$
\tilde{\varphi}\left(a_{0}, b_{0}\right)=-\int_{-1}^{1} \partial_{b} \tilde{\mathcal{G}}\left(a, 0, a_{0}, b_{0}\right) \tilde{\varphi}(a, 0) d a+\int_{-1}^{1} \tilde{\mathcal{G}}\left(a, 0, a_{0}, b_{0}\right) d a, \quad \forall\left(a_{0}, b_{0}\right) \in \tilde{\mathscr{O}}_{1}^{+} .
$$

We then introduce $\tilde{\mathscr{G}}$ defined by

$$
\tilde{\mathscr{G}}\left(a, a_{0}, b_{0}\right)=-\partial_{b} \tilde{\mathcal{G}}\left(a, 0, a_{0}, b_{0}\right)-\frac{1}{2 \pi} \frac{b_{0}}{b_{0}^{2}+\left(a-a_{0}\right)^{2}} .
$$

It is easily seen that for any $a_{0} \in(-1,1)$,

$$
\begin{aligned}
& \lim _{b_{0} \rightarrow 0} \int_{-1}^{1} \tilde{\mathscr{G}}\left(a, a_{0}, b_{0}\right) \tilde{\varphi}(a, 0) d a=\int_{-1}^{1} \tilde{\mathscr{G}}\left(a, a_{0}, 0\right) \tilde{\varphi}(a, 0) d a \\
& \lim _{b_{0} \rightarrow 0} \int_{-1}^{1} \tilde{\mathcal{G}}\left(a, 0, a_{0}, b_{0}\right) d a=\int_{-1}^{1} \tilde{\mathcal{G}}\left(a, 0, a_{0}, 0\right) d a
\end{aligned}
$$


whereas

$$
\lim _{b_{0} \rightarrow 0} \frac{1}{2 \pi} \int_{-1}^{1} \frac{b_{0}}{b_{0}^{2}+\left(a-a_{0}\right)^{2}} \tilde{\varphi}(a, 0) d a=\frac{1}{2} \tilde{\varphi}\left(a_{0}, 0\right) .
$$

Therefore, choosing $a_{0} \in(-1,1)$ and taking the limit $b_{0} \rightarrow 0$ in (3.18) leads to the following integral equation:

$$
\frac{1}{2} \tilde{\varphi}\left(a_{0}, 0\right)=\int_{-1}^{1} \tilde{\mathscr{G}}\left(a, a_{0}, 0\right) \tilde{\varphi}(a, 0) d a+\int_{-1}^{1} \tilde{\mathcal{G}}\left(a, 0, a_{0}, 0\right) d a .
$$

Discretizing equation (3.19), we can obtain a good approximation of $\tilde{\varphi}\left(a_{0}, 0\right)$ for $a_{0} \in(-1,1)$ (see Figure 3 ).

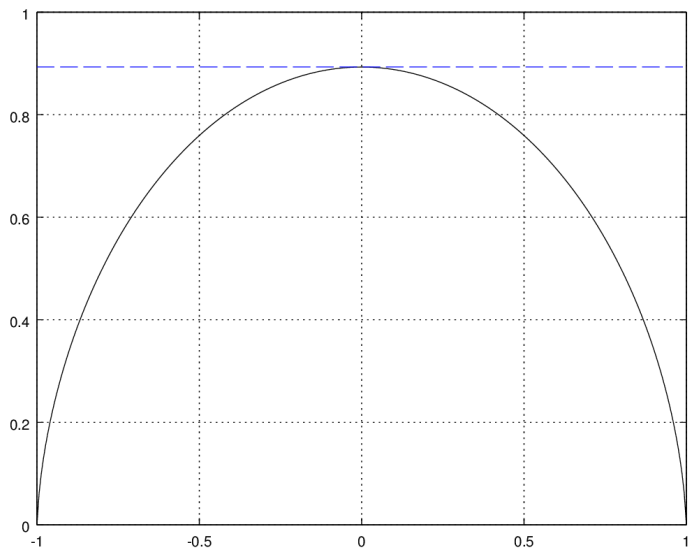

Figure 3: In solid black, $\tilde{\varphi}\left(a_{0}, 0\right)$ for $a_{0} \in(-1,1)$, obtained by discretization of equation (3.19).

In dashed blue, $\tilde{\Phi}(0,0)=\tilde{\varphi}(0,0)$.

\subsubsection{Phragmén Lindelöf principle}

With $\tilde{\varphi}$ as in 2.12 , the function $(a, b) \mapsto \tilde{\varphi}(a, b)+|b|$ is harmonic in $\tilde{\mathscr{O}}_{1}$, and it is therefore the real part of some holomorphic function $\phi$ in $\mathscr{O}_{1}$ :

$$
\forall(a, b) \in \tilde{\mathscr{O}}_{1}, \quad \Re(\phi(a+\imath b))=\tilde{\varphi}(a, b)+|b|,
$$

or, equivalently, for all $\xi \in \mathscr{O}_{1}, \Re(\phi(\xi))=\varphi(\xi)+|\Im(\xi)|$.

For each $\alpha_{*}>\alpha$, we consider the function $g_{\alpha_{*}}$ defined for $\xi \in \mathscr{O}_{\alpha}$ by

$$
g_{\alpha_{*}}(\xi)=f(\xi) \exp \left(-L \alpha_{*} \phi\left(\frac{\xi}{\alpha}\right)\right)
$$

By construction, $g_{\alpha_{*}}$ is holomorphic in $\mathscr{O}_{\alpha}$ and satisfies:

$$
\forall \xi \in \partial \mathscr{O}_{\alpha}, \quad\left|g_{\alpha_{*}}(\xi)\right| \leqslant C_{0}, \quad \text { and } \quad \lim _{|\Im(\xi)| \rightarrow \infty}\left(\sup _{|\Re(\xi)|<|\Im(\xi)|+\alpha}\left|g_{\alpha_{*}}(\xi)\right|\right)=0 .
$$

Therefore, $g_{\alpha_{*}}$ attains its maximum on $\partial \mathscr{O}_{\alpha}$, so that

$$
\forall \xi \in \mathscr{O}_{\alpha}, \quad|f(\xi)| \leqslant C_{0} \exp \left(\frac{\alpha_{*}}{\alpha}|\Im(\xi)| L\right) \exp \left(L \alpha_{*} \varphi\left(\frac{\xi}{\alpha}\right)\right) .
$$


Taking the limit $\alpha_{*} \rightarrow \alpha$, we immediately have

$$
\forall \xi \in \mathscr{O}_{\alpha}, \quad|f(\xi)| \leqslant C_{0} \exp (|\Im(\xi)| L) \exp \left(L \alpha \varphi\left(\frac{\xi}{\alpha}\right)\right)
$$

that is, 2.14.

Remark 3.2. Let us remark that we can obtain from the above proof an explicit formula for $f$. Indeed, for $\alpha_{*}>\alpha$, we can use the Cauchy formula for the function $g_{\alpha_{*}}$ in (3.20) on the contour given by

$$
\gamma_{\alpha, R}=\partial\left(\mathscr{O}_{\alpha} \cap\{\Im(\xi)<R\}\right), \quad(\text { with } R>0)
$$

oriented in a counter-clockwise manner, which yields: for all $\xi \in \mathbb{R}$ with $|\xi|<L /(2 T)$,

$$
g_{\alpha_{*}}(\xi)=\frac{1}{2 \imath \pi} \int_{\gamma_{\alpha, R}} \frac{g_{\alpha_{*}}(\zeta)}{\zeta-\xi} d \zeta
$$

Now, due to the decay of $g_{\alpha_{*}}$ at infinity, one can pass to the limit in the above formula as $R \rightarrow \infty:$ for all $\xi \in \mathbb{R}$ with $|\xi|<L /(2 T)$,

$$
g_{\alpha_{*}}(\xi)=\frac{1}{2 \imath \pi} \int_{\gamma_{\alpha}} \frac{g_{\alpha_{*}}(\zeta)}{\zeta-\xi} d \zeta
$$

where $\gamma_{\alpha}$ is the union of the two connected components of $\partial \mathscr{O}_{\alpha}$ oriented counter-clockwise. Recalling the definition of $g_{\alpha_{*}}$, we end up with the following formula: for all $\xi \in \mathbb{R}$ with $|\xi|<L /(2 T)$,

$$
f(\xi)=\frac{1}{2 \imath \pi} \int_{\gamma_{\alpha}} e^{L \alpha_{*}(\phi(\xi / \alpha)-\phi(\zeta / \alpha))} \frac{f(\zeta)}{\zeta-\xi} d \zeta
$$

\section{Further Comments}

\subsection{Higher dimensional settings}

The method developed above applies also to the cost of observability of the heat equation in multi-dimensional balls. More precisely, we consider the following heat equation, set in the ball of radius $L>0$ of $\mathbb{R}^{d}(d \geqslant 1)$, denoted by $\mathcal{B}_{L}$ in the following, and in the time interval $(0, T)$ :

$$
\begin{cases}\partial_{t} u-\Delta_{x} u=0, & \text { in }(0, T) \times \mathcal{B}_{L} \\ u(t, x)=0, & \text { in }(0, T) \times \partial \mathcal{B}_{L}, \\ u(0, x)=u_{0}(x), & \text { in } \mathcal{B}_{L}\end{cases}
$$

where the initial datum $u_{0}$ belongs to $H_{0}^{1}\left(\mathcal{B}_{L}\right)$. In that setting, we have the following result:

Theorem 4.1. Setting $K_{0}$ as in Theorem 1.1, for any $K>K_{0}$, there exists a constant $C>0$ such for all $T \in(0,1]$, for all solutions $u$ of 4.1 with initial datum $u_{0} \in H_{0}^{1}\left(\mathcal{B}_{L}\right)$,

$$
\left\|u(T) \exp \left(\frac{|x|^{2}}{4 T}\right)\right\|_{L^{2}\left(\mathcal{B}_{L}\right)} \leqslant C \exp \left(K \frac{L^{2}}{T}\right)\left\|\partial_{\nu} u\right\|_{L^{2}\left((0, T) \times \partial \mathcal{B}_{L}\right)} .
$$

Here and in the following, |.| denotes the euclidean norm in $\mathbb{R}^{d}$. The proof of Theorem 4.1 follows closely the one of Theorem 1.1. therefore we only sketch its proof, explaining the main differences with the proof of Theorem 1.1

Sketch of the proof of Theorem 4.1. We start by considering a smooth solution $u$ of (4.1), and define

$$
z(t, x)=u(t, x) \exp \left(\frac{|x|^{2}-L^{2}}{4 t}\right), \quad(t, x) \in(0, T) \times \mathcal{B}_{L},
$$

which satisfies

$$
\begin{cases}\partial_{t} z+\frac{x}{t} \cdot \nabla_{x} z+\frac{d}{2 t} z-\Delta_{x} z-\frac{L^{2}}{4 t^{2}} z=0 & \text { in }(0, \infty) \times \mathcal{B}_{L}, \\ z(t, x)=0 & \text { in }(0, T) \times \partial \mathcal{B}_{L}, \\ z(0, x)=0 & \text { in } \mathcal{B}_{L},\end{cases}
$$


Proposition A.1 with $\Omega=\mathcal{B}_{L}$ and $g \equiv 0$ implies directly the following estimate for $z$ :

$$
\int_{\mathcal{B}_{L}}\left|\nabla_{x} z(T, x)\right|^{2} d x-\frac{L^{2}}{4 T^{2}} \int_{\mathcal{B}_{L}}|z(T, x)|^{2} d x \leqslant \frac{L}{T^{2}} \int_{0}^{T} \int_{\partial \mathcal{B}_{L}} t\left|\nabla_{x} z(t, x) \cdot \nu\right|^{2} d s(x) d s .
$$

We define $w$ as the extension of $z$ by 0 outside $\mathcal{B}_{L}: w$ verifies the equations

$$
\begin{cases}\partial_{t} w+\frac{x}{t} \cdot \nabla_{x} w+\frac{d}{2 t} w-\Delta_{x} w-\frac{L^{2}}{4 t} w=\nabla_{x} u(t, x) \cdot \nu \delta_{\partial \mathcal{B}_{L}}, & \text { in }(0, \infty) \times \mathbb{R}^{d}, \\ w(0, x)=0, & x \in \mathbb{R}^{d} .\end{cases}
$$

Thus, its Fourier transform, defined for $(t, \xi) \in(0, T) \times \mathbb{C}^{d}$ by

$$
\widehat{w}(t, \xi)=\int_{\mathbb{R}^{d}} w(t, x) e^{-\imath \xi \cdot x} d x
$$

satisfies

$$
\begin{cases}\partial_{t} \widehat{w}-\frac{\xi}{t} \cdot \nabla_{\xi} \widehat{w}-\frac{d}{2 t} \widehat{w}+\xi^{2} \widehat{w}-\frac{L^{2}}{4 t^{2}} \widehat{w}=\int_{\partial \mathcal{B}_{L}} \nabla_{x} u(t, x) \cdot \nu e^{-\imath \xi \cdot x} d s(x), & (t, \xi) \in(0, \infty) \times \mathbb{R}^{d}, \\ \widehat{w}(0, \xi)=0, & \xi \in \mathbb{R}^{d} .\end{cases}
$$

As in the one-dimensional case, equation (4.3) gives a high-frequency $(|\xi|>L /(2 T)) L^{2}$-estimate of $w(T, \cdot)$ depending on the observation and the low-frequency $(|\xi| \leqslant L /(2 T)) L^{2}$-norm of $w(T, \cdot)$, on which we focus from now. To do so, similarly as in Section 3.1, we solve the transport equation (4.4), and obtain, for $\xi_{0} \in \mathbb{R}^{d}$ such that $\left|\xi_{0}\right|>L /(2 T)$,

$$
\widehat{w}\left(T, \xi_{0}\right)=\int_{0}^{T}\left(\frac{T}{t}\right)^{\frac{d}{2}} \int_{\partial \mathcal{B}_{L}} \nabla_{x} u(t, x) \cdot \nu e^{-\imath \frac{x \cdot \xi_{0} T}{t}-\left(\xi_{0}^{2} T^{2}-L^{2} / 4\right)(1 / t-1 / T)} d s(x) d t
$$

with $\xi_{0}^{2}=\xi_{0} \cdot \xi_{0}$.

Once here, we consider $\xi_{0}=\left(\xi_{1}, \tilde{\xi}\right)$, with $\tilde{\xi} \in \mathbb{R}^{d-1}$ fixed, and $\xi_{1}=a+\imath b, a, b \in \mathbb{R}$, and define $f\left(\xi_{1}\right)=$ $\widehat{w}\left(T, \xi_{1}, \tilde{\xi}\right)$ which is an entire function satisfying (2.11). Besides, with similar computations as in Section 3.1. it is easy to obtain that for all $\alpha>L^{2} /(2 T)$, there exists $C_{\alpha}(T)>0$, which may blow up polynomially in $T$ as $T \rightarrow 0$ (contrarily to what happens in the one-dimensional setting, the constant $C_{\alpha}(T)$ may now blow up as $T \rightarrow 0$, but only polynomially in $T$, so that it will not significantly affect the cost of observability in small times in (4.2), which blows up as an exponential of $1 / T$ as $T \rightarrow 0$ ), such that for all $\xi_{1} \in \mathscr{C}_{\alpha}$ as in (2.6), we have

$$
\left|f\left(\xi_{1}\right)\right| \leqslant C_{\alpha} e^{\left|\Im\left(\xi_{1}\right)\right| L}\left\|\partial_{\nu} u\right\|_{L^{2}\left((0, T) \times \partial \mathcal{B}_{L}\right)} .
$$

From that, we end the proof of Theorem 4.1 exactly as in the one-dimensional case, with the use of Proposition 2.3

Actually, the method developed above works not only for balls, but also for any bounded domain $\Omega \subset \mathbb{R}^{d}$. More precisely:

Theorem 4.2. Let $\Omega$ be a smooth bounded domain of $\mathbb{R}^{d}$, if we set

$$
L_{\Omega}=\inf _{x \in \Omega} \sup _{y \in \partial \Omega}|x-y|
$$

and we choose $\bar{x} \in \bar{\Omega}$ such that

$$
\sup _{y \in \partial \Omega}|\bar{x}-y|=L_{\Omega}
$$

Then for any $K>K_{0}$, there exists $C>0$ such that any smooth function $u$ solution of

verifies

$$
\begin{cases}\partial_{t} u-\Delta_{x} u=0 & \text { in }(0, T) \times \Omega \\ u(t, x)=0 & \text { in }(0, T) \times \partial \Omega \\ u(0, x)=u_{0}(x) & \text { in } \Omega\end{cases}
$$

$$
\left\|u(T) \exp \left(\frac{|x-\bar{x}|^{2}}{4 T}\right)\right\|_{L^{2}(\Omega)} \leqslant C \exp \left(K L_{\Omega}^{2} / T\right)\left\|\partial_{\nu} u\right\|_{L^{2}((0, T) \times \partial \Omega)} .
$$


Note that this is a geometrical setting in which Corollary 1.3 applies but yields a different estimate on the cost of observability. Indeed, when the observation is done on the whole boundary, one easily checks that the choice $S_{0}=S_{\Omega}^{+}$, where

$$
S_{\Omega}=\sup \{\text { Length of segments included in } \Omega\},
$$

is suitable for the application of Corollary 1.3 In particular, when $\Omega$ is convex, $L_{\Omega} \leqslant S_{\Omega} \leqslant 2 L_{\Omega}$ and Theorem 4.2 always yields at least the estimate given by Corollary 1.3 when the observation is done on the whole boundary of $\Omega$, and a better one in general (as in the case of a ball discussed in Theorem 4.1).

\subsection{Tensorized equations}

Another application of our method concerns the cost of observability of the heat equation on a tensorized domain. More precisely, we consider the heat equation set in a tensorized spatial domain $\Omega=\Omega_{x} \times \Omega_{y}$, and want to know the cost of observability in small time when the solution is observed on $\partial \Omega_{x} \times \Omega_{y}$. Note that the answer is already known: the cost is the same as the one for the heat equation set on $\Omega_{x}$ only, when the observation is done on the whole boundary $\partial \Omega_{x}$ [33. Theorem 1.5]. Our purpose is therefore just to underline that our approach also applies in that context and allows to retrieve easily this result.

To fix ideas, we focus on the case $\Omega_{x}=(-L, L)$ (When $\Omega_{x}$ is a multi-dimensional domain, similar arguments can be developed, under appropriate geometric conditions, by using Theorem 4.2 instead of Theorem 1.1). Hence we are interested in the following heat equation, set in the domain $\Omega=(-L, L) \times \Omega_{y}$, with $L>0$ and $\Omega_{y}$ a smooth bounded domain of $\mathbb{R}^{d_{y}}$, in some time interval $(0, T), T>0$ :

$$
\begin{cases}\partial_{t} u-\partial_{x}^{2} u-\Delta_{y} u=0 & \text { for }(t, x, y) \in(0, T) \times(-L, L) \times \Omega_{y} \\ u(t, L, y)=u(t,-L, y)=0 & \text { for }(t, y) \in(0, T) \times \Omega_{y} \\ u(t, x, y)=0 & \text { for }(t, x, y) \in(0, T) \times(-L, L) \times \partial \Omega_{y} \\ u(0, x, y)=u_{0}(x, y) & \text { in }(-L, L) \times \Omega_{y}\end{cases}
$$

As usual, the initial datum $u_{0}$ belongs to $H_{0}^{1}\left((-L, L) \times \Omega_{y}\right)$. We have the following:

Theorem 4.3. Setting $K_{0}$ as in Theorem 1.1, for any $K>K_{0}$, there exists a constant $C>0$ such for all $T \in(0,1]$, for all solutions $u$ of 4.6 ,

$$
\begin{aligned}
\| u(T, x, y) \exp \left(\frac{x^{2}}{4 T}\right) & \|_{L^{2}\left((-L, L) \times \Omega_{y}\right)} \\
& \leqslant C \exp \left(K \frac{L^{2}}{T}\right)\left(\left\|\partial_{x} u(t,-L, y)\right\|_{L^{2}\left((0, T) \times \Omega_{y}\right)}+\left\|\partial_{x} u(t, L, y)\right\|_{L^{2}\left((0, T) \times \Omega_{y}\right)}\right) .
\end{aligned}
$$

Sketch of the proof of Theorem 4.3. Let us denote by $\left(v_{n}, \lambda_{n}^{2}\right)$ the family of normalized eigenfunctions and eigenvalues of the Dirichlet-Laplace operator set in $\Omega_{y}$, that is

$$
\left\{\begin{aligned}
-\Delta_{y} v_{n} & =\lambda_{n}^{2} v_{n} & & \text { in } \Omega_{y}, \\
v_{n} & =0 & & \text { on } \partial \Omega_{y}, \\
\left\|v_{n}\right\|_{L^{2}\left(\Omega_{y}\right)} & =1 . & &
\end{aligned}\right.
$$

Expanding $u$ solution of 4.6 on the $L^{2}\left(\Omega_{y}\right)$ Hilbert basis $\left(v_{n}\right)$, that is

$$
u(t, x, y)=\sum_{n \in \mathbb{N}} u_{n}(t, x) v_{n}(y)
$$

we see that each $u_{n}$ solves a one dimensional heat equation with potential $\lambda_{n}^{2}$ set in $(0, T) \times(-L, L)$ :

$$
\begin{cases}\partial_{t} u_{n}-\partial_{x}^{2} u_{n}+\lambda_{n}^{2} u_{n}=0 & \text { in }(0, T) \times(-L, L) \\ u_{n}(t,-L)=u_{n}(t, L)=0 & \text { in }(0, T) \\ u_{n}(0, x)=u_{n, 0}(x) & \text { in }(-L, L)\end{cases}
$$

with

$$
u_{n, 0}(x)=\int_{\Omega} u_{0}(x, y) v_{n}(y) d y
$$


To prove Theorem 4.3 , it is sufficient to prove that each $u_{n}$ verifies the following observability inequality

$$
\left\|u_{n}(T, x) \exp \left(\frac{x^{2}}{4 T}\right)\right\|_{L^{2}(-L, L)} \leqslant C \exp \left(K \frac{L^{2}}{T}\right)\left(\left\|\partial_{x} u_{n}(t,-L)\right\|_{L^{2}(0, T)}+\left\|\partial_{x} u_{n}(t, L)\right\|_{L^{2}(0, T)}\right),
$$

with a constant $C$ independent of $n$. To do so, we consider $\tilde{u}_{n}=u_{n} e^{\lambda_{n}^{2} t}$, which verifies

$$
\begin{cases}\partial_{t} \tilde{u}_{n}-\partial_{x}^{2} \tilde{u}_{n}=0 & \text { in }(0, T) \times(-L, L) \\ \tilde{u}_{n}(t,-L)=\tilde{u}_{n}(t, L)=0 & \text { in }(0, T) \\ \tilde{u}_{n}(0, x)=u_{n, 0}(x) & \text { in }(-L, L)\end{cases}
$$

Applying Theorem 1.1 , we get

$$
\left\|\tilde{u}_{n}(T, x) \exp \left(\frac{x^{2}}{4 T}\right)\right\|_{L^{2}(-L, L)} \leqslant C \exp \left(K \frac{L^{2}}{T}\right)\left(\left\|\partial_{x} \tilde{u}_{n}(t,-L)\right\|_{L^{2}(0, T)}+\left\|\partial_{x} \tilde{u}_{n}(t, L)\right\|_{L^{2}(0, T)}\right),
$$

which directly gives 4.9 as $e^{\lambda_{n}^{2}(t-T)} \leqslant 1$ for all $t \in(0, T)$, and therefore ends the proof.

\subsection{Observation from one side of the domain - Symmetrization argument}

In this section, we are interested in the cost of observability for the one dimensional heat equation when observed on one side of the domain. In other words, for $L, T>0$ and $u_{0} \in H_{0}^{1}(0, L)$, we consider the system

$$
\begin{cases}\partial_{t} u-\partial_{x}^{2} u=0, & \text { in }(0, T) \times(0, L) \\ u(t, 0)=u(t, L)=0, & \text { in }(0, T) \\ u(0, x)=u_{0}(x), & \text { in }(0, L)\end{cases}
$$

We have the following:

Theorem 4.4. Setting $K_{0}$ as in Theorem 1.1, for any $K>K_{0}$, there exists a constant $C>0$ such for all $T \in(0,1]$, for all solutions $u$ of 4.10 with $u_{0} \in H_{0}^{1}(0, L)$,

$$
\left\|u(T) \exp \left(\frac{x^{2}}{4 T}\right)\right\|_{L^{2}(0, L)} \leqslant C \exp \left(K \frac{L^{2}}{T}\right)\left\|\partial_{x} u(t, L)\right\|_{L^{2}(0, T)} .
$$

Proof. The proof is based on a classical symmetrisation argument: for $u$ solution of 4.10 , we define

$$
u_{s}(t, x)=\left\{\begin{array}{cl}
u(t, x) & \text { for }(t, x) \in(0, T) \times(0, L) \\
-u(t,-x) & \text { for }(t, x) \in(0, T) \times(-L, 0) .
\end{array}\right.
$$

It is readily seen that $u_{s}$ verifies system 1.1. Therefore, Theorem 1.1 gives

$$
\left\|u_{s}(T) \exp \left(\frac{x^{2}}{4 T}\right)\right\|_{L^{2}(-L, L)} \leqslant C \exp \left(\frac{K L^{2}}{T}\right)\left(\left\|\partial_{x} u_{s}(t,-L)\right\|_{L^{2}(0, T)}+\left\|\partial_{x} u_{s}(t, L)\right\|_{L^{2}(0, T)}\right) .
$$

The result follows easily, as $\partial_{x} u_{s}(t,-L)=\partial_{x} u_{s}(t, L)=\partial_{x} u(t, L)$ for all $t \in(0, T)$.

\subsection{Distributed observations}

One is sometimes interested in distributed observations, in which case the corresponding observability inequality reads:

$$
\|u(T)\|_{L^{2}(0, L)} \leqslant C(T, L, a, b)\|u\|_{L^{2}((0, T) \times(a, b))},
$$

for smooth solutions $u$ of 4.10 , where $a, b \in \mathbb{R}$ are such that $(a, b) \subset(0, L)$ and $a<b$.

We claim the following:

Theorem 4.5. Let $0 \leqslant a<b \leqslant L$. Setting $K_{0}$ as in Theorem 1.1, for any $K>K_{0}$, there exists a constant $C>0$ such for all $T \in(0,1]$, for all solutions $u$ of 4.10$)$,

$$
\|u(T)\|_{L^{2}(0, L)} \leqslant C \exp \left(\frac{K \min \left\{a^{2},(L-b)^{2}\right\}}{T}\right)\|u\|_{L^{2}\left(0, T ; H^{1}(a, b)\right)} .
$$


Proof. As in the proof of Theorem 4.4, we start by symmetrizing the function $u$, and we call $u_{s}$ its symmetric extension. We then take $\varepsilon>0$ small enough to have $a+2 \varepsilon<b$ and we choose an even cut-off function $\rho$ taking value 1 on $(-a-\varepsilon, a+\varepsilon)$ and vanishing for $|x|>a+2 \varepsilon$. Then the function

$$
z(t, x)= \begin{cases}\rho(x) u_{s}(t, x) \exp \left(\frac{x^{2}-(a+2 \varepsilon)^{2}}{4 t}\right) & \text { for }|x|<a+2 \varepsilon, \\ 0 & \text { for }|x|>a+2 \varepsilon\end{cases}
$$

satisfies, similarly as in 2.3 ,

$$
\begin{cases}\partial_{t} z+\frac{x}{t} \partial_{x} z+\frac{1}{2 t} z-\partial_{x}^{2} z-\frac{(a+2 \varepsilon)^{2}}{4 t^{2}} z=g, & (t, x) \in(0, \infty) \times(-a-2 \varepsilon, a+2 \varepsilon), \\ z(t,-a-2 \varepsilon)=z(t, a+2 \varepsilon)=0, & t \in(0, \infty), \\ z(0, x)=0, & x \in(-a-2 \varepsilon, a+2 \varepsilon),\end{cases}
$$

where

$$
g(t, x)=\exp \left(\frac{x^{2}-(a+2 \varepsilon)^{2}}{4 t}\right)\left(2 \partial_{x} \rho \partial_{x} u(t, x)+\partial_{x x} \rho u(t, x)\right) .
$$

One can then follow the approach developed in Section 2 (using Proposition A.1 instead of Theorem 2.1 and the fact that $\left.\partial_{x} z(t,-a-2 \varepsilon)=\partial_{x} z(t, a+2 \varepsilon)=0\right)$ to show that for all $K_{1}>K_{0}$, there exists $C$ such that for all $T \in(0,1]$,

$$
\|z(T)\|_{L^{2}(-a-2 \varepsilon, a+2 \varepsilon)} \leqslant C \exp \left(\frac{K_{1}(a+2 \varepsilon)^{2}}{T}\right)\|g\|_{L^{2}((0, T) \times(-a-2 \varepsilon, a+2 \varepsilon))} .
$$

Using the definition of $z$ and $g$, one easily gets

$$
\|u(T)\|_{L^{2}(0, a+\varepsilon)} \leqslant C \exp \left(\frac{K_{1}(a+2 \varepsilon)^{2}}{T}\right)\|u\|_{L^{2}\left(0, T ; H^{1}(a, a+2 \varepsilon)\right)} .
$$

Similarly, one can obtain

$$
\|u(T)\|_{L^{2}(b-\varepsilon, L)} \leqslant C \exp \left(\frac{K_{1}(L-b+2 \varepsilon)^{2}}{T}\right)\|u\|_{L^{2}\left(0, T ; H^{1}(b-2 \varepsilon, b)\right)} .
$$

It is besides straightforward to show that

$$
\|u(T)\|_{L^{2}(a+\varepsilon, b-\varepsilon)} \leqslant C\|u\|_{L^{2}\left(0, T ; H^{1}(a, b)\right)},
$$

for instance by looking at $v(t, x)=\eta(t) u(t, x) \rho_{0}(x)$, where $\eta=\eta(t)$ is a smooth function of time taking value 0 at $t=0$ and 1 at $t=T$, and $\rho_{0}=\rho_{0}(x)$ taking value 1 on $(a+\varepsilon, b-\varepsilon)$ and vanishing for $x \notin(a, b)$, and doing energy estimates.

Combining the three above estimates, we easily conclude 4.13 by taking $K_{1} \in\left(K_{0}, K\right)$ and $\varepsilon>0$ small enough.

Note that the above argument is only based on suitable cut-off arguments. It can therefore be applied as well in multi-dimensional settings, provided some geometric assumptions compatible with Theorem 4.2 are satisfied, namely if the distributed observation set is a neighborhood of the whole boundary.

\subsection{Related uncertainty principles}

One key point to obtain Theorem 1.1 is the complex analysis argument developed in Section 3.2 , based principally on the Schwarz-Christoffel conformal mapping and the Phragmén Lindelöf principle. It is nevertheless possible to develop a purely real analysis argument, but it only allows to retrieve the cost of observability for the one-dimensional heat equation known since [37:

Theorem 4.6. For all $K>3 / 4$, there exists a constant $C>0$ such for all $T \in(0,1]$, all solutions $u$ of (1.1) with initial datum $u_{0} \in H_{0}^{1}(-L, L)$ satisfies 1.2 . 
The proof of Theorem 4.6 is based on the following uncertainty principle result, due to [24, 13]:

Proposition 4.7 (24, 13]). Let $A, B>0$. Let $f \in L^{2}(\mathbb{R})$ supported in $[-A, A], \widehat{f}$ its Fourier transform. Then

$$
\int_{-B}^{B}|\widehat{f}(\xi)|^{2} d \xi \leqslant \lambda_{0} \int_{\mathbb{R}}|\widehat{f}(\xi)|^{2} d \xi
$$

where $\lambda_{0}=\lambda_{0}(A B)$ verifies $0<\lambda_{0}<1$ and

$$
\lambda_{0}=1-4 \sqrt{\pi} \sqrt{A B} e^{-2 A B}\left(1+\epsilon_{A B}\right),
$$

where $\epsilon_{A B} \rightarrow 0$ as $A B \rightarrow \infty$.

Relation 4.15 is a particular case of [24, Theorem p.68], whereas the proof of the asymptotic behaviour of $\lambda_{0}$ can be found in [13. Theorem 1, p.319].

Proof of Theorem 4.6. We start from formula 2.7), which we recall hereafter: for any $\xi_{0} \in \mathbb{R}$ such that $\left|\xi_{0}\right|>L /(2 T)$, we have

$$
\begin{aligned}
\widehat{w}\left(T, \xi_{0}\right)=-\int_{0}^{T} \sqrt{\frac{T}{t}} \partial_{x} u(t,-L) e^{\imath \frac{L \xi_{0} T}{t}-\left(\xi_{0}^{2} T^{2}-L^{2} / 4\right)(1 / t-1 / T)} d t & \\
& +\int_{0}^{T} \sqrt{\frac{T}{t}} \partial_{x} u(t, L) e^{-\imath \frac{L \xi_{0} T}{t}-\left(\xi_{0}^{2} T^{2}-L^{2} / 4\right)(1 / t-1 / T)} d t .
\end{aligned}
$$

Therefore, we directly obtain, for $\xi_{0} \in \mathbb{R}$ with $\left|\xi_{0}\right|>L /(2 T)$,

$$
\left|\widehat{w}\left(T, \xi_{0}\right)\right|^{2} \leqslant T\left(\left\|\partial_{x} u(t,-L)\right\|_{L^{2}(0, T)}^{2}+\left\|\partial_{x} u(t, L)\right\|_{L^{2}(0, T)}^{2}\right) \int_{0}^{T} e^{-2 T^{2}\left(\xi_{0}^{2}-L^{2} / 4 T^{2}\right)(1 / t-1 / T)} \frac{d t}{t} .
$$

For $\eta>1$, we choose $\xi_{0} \in \mathbb{R}$ with $\left|\xi_{0}\right| \geqslant \eta L /(2 T)$ which implies

$$
\xi_{0}^{2}-\frac{L^{2}}{4 T^{2}} \geqslant \frac{\eta^{2}-1}{\eta^{2}} \xi_{0}^{2}
$$

and

$$
\int_{0}^{T} e^{-2 T^{2}\left(\xi_{0}^{2}-L^{2} / 4 T^{2}\right)(1 / t-1 / T)} \frac{d t}{t} \leqslant \int_{0}^{T} e^{-2 T^{2} \frac{\eta^{2}-1}{\eta^{2}} \xi_{0}^{2}\left(\frac{1}{t}-\frac{1}{T}\right)} \frac{d t}{t} \leqslant \frac{\eta^{2}}{2 T\left(\eta^{2}-1\right) \xi_{0}^{2}} .
$$

Hence we obtain, for $\xi_{0} \in \mathbb{R}$ with $\left|\xi_{0}\right|>L /(2 T)$,

$$
\left|\widehat{w}\left(T, \xi_{0}\right)\right|^{2} \leqslant \frac{\eta^{2}}{2\left(\eta^{2}-1\right) \xi_{0}^{2}}\left(\left\|\partial_{x} u(t,-L)\right\|_{L^{2}(0, T)}^{2}+\left\|\partial_{x} u(t, L)\right\|_{L^{2}(0, T)}^{2}\right)
$$

and

$$
\int_{\left|\xi_{0}\right|>\eta \frac{L}{2 T}}\left|\widehat{w}\left(T, \xi_{0}\right)\right|^{2} d \xi_{0} \leqslant \frac{2 T \eta}{\left(\eta^{2}-1\right) L}\left(\left\|\partial_{x} u(t,-L)\right\|_{L^{2}(0, T)}^{2}+\left\|\partial_{x} u(t, L)\right\|_{L^{2}(0, T)}^{2}\right) .
$$

Now, from 4.15 applied to $f=\widehat{w}(T)$ with $A=L, B=\eta L /(2 T)$ and $\lambda_{0}=\lambda_{0}\left(\eta L^{2} /(2 T)\right)$, we have

$$
\begin{aligned}
\int_{\mathbb{R}}\left|\widehat{w}\left(T, \xi_{0}\right)\right|^{2} d \xi_{0} & =\int_{\left|\xi_{0}\right|<\eta \frac{L}{2 T}}\left|\widehat{w}\left(T, \xi_{0}\right)\right|^{2} d \xi_{0}+\int_{\left|\xi_{0}\right|>\eta \frac{L}{2 T}}\left|\widehat{w}\left(T, \xi_{0}\right)\right|^{2} d \xi_{0} \\
& \leqslant \lambda_{0} \int_{\mathbb{R}}\left|\widehat{w}\left(T, \xi_{0}\right)\right|^{2} d \xi_{0}+\int_{\left|\xi_{0}\right|>\eta \frac{L}{2 T}}\left|\widehat{w}\left(T, \xi_{0}\right)\right|^{2} d \xi_{0},
\end{aligned}
$$

and thus

We have thus obtained

$$
\int_{\mathbb{R}}\left|\widehat{w}\left(T, \xi_{0}\right)\right|^{2} d \xi_{0} \leqslant \frac{1}{1-\lambda_{0}} \int_{\left|\xi_{0}\right|>\eta \frac{L}{2 T}}\left|\widehat{w}\left(T, \xi_{0}\right)\right|^{2} d \xi_{0}
$$

$$
\int_{-L}^{L}|w(T, x)|^{2} d x=\int_{\mathbb{R}}\left|\widehat{w}\left(T, \xi_{0}\right)\right|^{2} d \xi_{0} \leqslant \frac{1}{1-\lambda_{0}} \frac{2 T \eta}{\left(\eta^{2}-1\right) L}\left(\left\|\partial_{x} u(t,-L)\right\|_{L^{2}(0, T)}^{2}+\left\|\partial_{x} u(t, L)\right\|_{L^{2}(0, T)}^{2}\right)
$$


which implies from Proposition 4.7 and 4.16 the existence of a constant $C$ such that for $T$ small enough

$$
\int_{-L}^{L}|w(T, x)|^{2} d x \leqslant C e^{\eta \frac{L^{2}}{T}}\left(\left\|\partial_{x} u(t,-L)\right\|_{L^{2}(0, T)}^{2}+\left\|\partial_{x} u(t, L)\right\|_{L^{2}(0, T)}^{2}\right) .
$$

The result of Theorem 4.6 follows from the definition of $w$.

\subsection{On a possible improvement of Theorem 1.1}

As we said in the introduction, we do not know if the estimate on the cost of observability in small times given by Theorem 1.1 is sharp or not. In fact, when looking at the main steps of the proof of Theorem 1.1 given in Section 2, it seems that one step in which our estimates are not sharp may be the one using Phragmén-Lindelöf principles, i.e. Proposition 2.3

Indeed, introducing the class

$$
\mathscr{E}_{\alpha}=\left\{f \in \operatorname{Hol}\left(\mathcal{O}_{\alpha}\right) \text {, s.t. } f(\xi) e^{-|\Im(\xi)|} \in L^{\infty}\left(\mathscr{O}_{\alpha}\right) \text { and } \forall \xi \in \partial \mathcal{O}_{\alpha}, \quad|f(\xi)| \leqslant e^{|\Im(\xi)|}\right\},
$$

Proposition 2.3 shows that

$$
\sup _{f \in \mathscr{E}_{\alpha}}\left(\sup _{x \in[-\alpha, \alpha]}\{|f(x)|\}\right) \leqslant \exp (\alpha \varphi(0)),
$$

where $\varphi(0)$ is given by 2.15. Besides, this estimate is sharp as we can construct an holomorphic function $\phi$ in $\mathscr{O}_{1}$ whose real part coincides with $\varphi(\xi)+|\Im(\xi)|$ given by $2.12-2.13$ and check that $f_{\phi}(\xi)=\exp (\alpha \phi(\xi / \alpha))$ belongs to $\mathscr{E}_{\alpha}$ and saturates the estimate 4.17 , so that

$$
\max _{f \in \mathscr{E}_{\alpha}}\left(\max _{x \in[-\alpha, \alpha]}\{|f(x)|\}\right)=\exp (\alpha \varphi(0))
$$

Now, in our approach (in the case $L=1$, which can always be assumed by a scaling argument), we apply estimate 4.17) to the function $f=\widehat{w}(T, \cdot) /\left\|\widehat{w}(T, \xi) e^{-|\Im(\xi)|}\right\|_{L^{\infty}\left(\mathscr{C}_{\alpha}\right)}$, which in fact belongs to a smaller class:

$$
\mathscr{E}_{\alpha}^{*}=\left\{f \in \operatorname{Hol}(\mathbb{C}), \text { s.t. } f(\xi) e^{-|\Im(\xi)|} \in L^{\infty}(\mathbb{C}) \text { and } \forall \xi \in \mathscr{C}_{\alpha}, \quad|f(\xi)| \leqslant e^{|\Im(\xi)|} .\right\}
$$

Therefore, our proof requires an estimate on the constant

$$
C^{*}(\alpha)=\sup _{f \in \mathscr{E}_{\alpha}^{*}}\left(\sup _{x \in[-\alpha, \alpha]}\{|f(x)|\}\right),
$$

in the asymptotics $\alpha \rightarrow \infty$. It is clear that

$$
C^{*}(\alpha) \leqslant \exp (\alpha \varphi(0))
$$

which is precisely the estimate we use, but there is no evidence to support the idea that this estimate gives the good asymptotics as $\alpha \rightarrow \infty$.

Let us in particular point out that

- The function $f_{\phi}$ given above to show that estimate 4.17) is sharp does not belong to the class $\mathscr{E}_{\alpha}^{*}$.

- The constant $C^{*}(\alpha)$ in 4.19 blows up at least like $\exp (\alpha / 2)$ as $\alpha \rightarrow \infty$, as otherwise the proof given in Section 2 would yield a cost of observability smaller than $\exp \left(L^{2} / 2 T\right)$ in small times, which is known to be false due to 29 .

- Looking at the 2-parameters family of functions of the form

$$
f_{A, \gamma}(\xi)=\cos \left(A \sqrt{\xi^{2}-\gamma^{2}}\right)
$$

for parameters $A \in[0,1]$ and $\gamma \in[0, \alpha]$, we find out that

$$
\sup _{f \in\left\{f_{A, \gamma}\right\} \cap \mathscr{E}_{\alpha}^{*}}\left(\sup _{x \in[-\alpha, \alpha]}\{|f(x)|\}\right)=\cosh \left(\frac{\alpha}{2}\right),
$$


and is achieved when taking $A=1 / \sqrt{2}$ and $\gamma=\alpha / \sqrt{2}$, i.e.

$$
f(\xi)=\cos \left(\frac{1}{\sqrt{2}} \sqrt{\xi^{2}-\frac{\alpha^{2}}{2}}\right) .
$$

This function yields another evidence of the fact that

$$
\liminf _{\alpha \rightarrow \infty} \log \left(C^{*}(\alpha)\right) \geqslant \frac{\alpha}{2} .
$$

Let us finally emphasize that if we were able to show that

$$
\limsup _{\alpha \rightarrow \infty} \log \left(C^{*}(\alpha)\right) \leqslant \frac{\alpha}{2}
$$

the proof given in Section 2 would yield a cost of observability in small times $C_{0}(T, L)$ satisfying

$$
\limsup _{\alpha \rightarrow \infty} T \log \left(C_{0}(T, L)\right) \leqslant \frac{L^{2}}{2} .
$$

Combined with 29], this would entail that

$$
\lim _{\alpha \rightarrow \infty} T \log \left(C_{0}(T, L)\right)=\frac{L^{2}}{2}
$$

\subsection{Uniform controllability of viscous approximations of the transport equation}

The problem we considered in this article is intimately related to the question of uniform controllability of viscous approximations of the transport equation raised in [4]. Namely, for all $\varepsilon>0$, one considers the following viscous approximation of the transport equation at velocity $M \in \mathbb{R}$ :

$$
\begin{cases}\partial_{t} y_{\varepsilon}-\varepsilon \partial_{x}^{2} y_{\varepsilon}+M \partial_{x} y_{\varepsilon}=0, & (t, x) \in(0, T) \times(0, L), \\ y_{\varepsilon}(t, 0)=v_{\varepsilon}(t), & t \in(0, T), \\ y_{\varepsilon}(t, L)=0, & t \in(0, T), \\ y_{\varepsilon}(0, \cdot)=y_{0}(x), & x \in(0, L)\end{cases}
$$

For each $\varepsilon>0$, the equation 4.21) is null-controllable in any time $T>0$, and the map $\mathcal{V}_{\varepsilon, T}: y_{0} \rightarrow v_{\varepsilon}$ which to any $y_{0} \in L^{2}(0, L)$ associates the control $v_{\varepsilon}$ of minimal $L^{2}(0, T)$-norm is linear. The question raised in [4] is the following one: Give conditions on the time $T$ guaranteeing that

$$
\limsup _{\varepsilon \rightarrow 0}\left\|\mathcal{V}_{\varepsilon, T}\right\|_{\mathscr{L}\left(L^{2}(0, L) ; L^{2}(0, T)\right)}<\infty
$$

It is clear that if $|M| T<L, \sqrt{4.22}$ cannot happen, as otherwise the convergence of (4.21) as $\varepsilon \rightarrow 0$ would imply the null-controllability of the transport equation in a time which is not enough to make the characteristics go out of the domain.

Several conditions on the time $T$ ensuring 4.22 were then proposed in the literature, namely in the works [4], 17] and [28. In fact, to our knowledge, the best results are the ones obtained in [28, which we recall now:

Theorem 4.8 ([28]). If $M \neq 0$ and

$$
|M| T>T(2 \sqrt{3}+1-\operatorname{sign}(M)), \quad(2 \sqrt{3} \approx 3.4641),
$$

where $\operatorname{sign}(M)=1$ if $M>0$ and $=-1$ if $M<0$, we have

$$
\limsup _{\varepsilon \rightarrow 0}\left\|\mathcal{V}_{\varepsilon, T}\right\|_{\mathscr{L}\left(L^{2}(0, L) ; L^{2}(0, T)\right)}=0 .
$$


These results are based on the knowledge of the cost of observability of the one-dimensional heat equation in small time obtained in [37. Therefore, as Theorem 4.4 improves the one in [37, following the proof of [28. immediately improves the known result on the uniform controllability of the viscous approximations 4.21) of the transport equation:

Theorem 4.9. Let $K_{0}$ as in 1.5 . Then, if $M \neq 0$ and

$$
|M| T>L\left(4 \sqrt{K_{0}}+1-\operatorname{sign}(M)\right), \quad\left(4 \sqrt{K_{0}} \approx 3.3385\right),
$$

we have

$$
\limsup _{\varepsilon \rightarrow 0}\left\|\mathcal{V}_{\varepsilon, T}\right\|_{\mathscr{L}\left(L^{2}(0, L) ; L^{2}(0, T)\right)}=0 .
$$

As the proof of Theorem 4.9 follows line to line the one of [28, it is left to the reader.

We are currently investigating if one can do better than the combination of the cost of observability of the one-dimensional heat equation in small times and of the arguments in 28 to obtain better sufficient conditions on the ratio $|M| T / L$ to guarantee 4.23 . We believe that a direct approach following the strategy in Section 2 could help improving Theorem 4.9

\section{A Carleman-type estimate}

We consider the following equation

$$
\begin{cases}\partial_{t} z-\Delta_{x} z+\frac{1}{2 t}\left(2 x \cdot \nabla_{x} z+d z\right)-\frac{L^{2}}{4 t^{2}} z=g & \text { in }(0, T) \times \Omega, \\ z(t, x)=0 & \text { on }(0, T) \times \partial \Omega \\ \lim _{t \rightarrow 0}\|z(t)\|_{L^{2}(\Omega)}=0 & \\ \lim _{t \rightarrow 0} t\|\nabla z(t)\|_{L^{2}(\Omega)}=0\end{cases}
$$

with $T>0, \Omega$ a bounded domain of $\mathbb{R}^{d}, d \geqslant 1$,

$$
L=\sup _{x \in \Omega}|x| .
$$

and

We then have the following result:

$$
g \in L^{2}((0, T) \times \Omega)
$$

Proposition A.1. Any smooth solution $z$ of A.1 with $g \in L^{2}((0, T) \times \Omega)$ verifies the following estimate:

$$
\int_{\Omega}\left(\left|\nabla_{x} z(T)\right|^{2}-\frac{L^{2}}{4 T^{2}}|z(T)|^{2}\right) d x \leqslant \frac{L}{T^{2}} \int_{0}^{T}\left(t \int_{\Gamma_{+}}\left|\nabla_{x} z(t, x) \cdot \nu\right|^{2} d s(x)\right) d t+\frac{1}{T^{2}} \int_{0}^{T} \int_{\Omega} t^{2}|g|^{2} d x d t
$$

with $\Gamma_{+}=\{x \in \partial \Omega, x \cdot \nu>0\}$, and $L$ is given by $\mathrm{A} .2$.

Proof. We define the following spatial operators

$$
S z=-\Delta_{x} z-\frac{L^{2}}{4 t^{2}} z, \quad A z=\frac{1}{2 t}\left(2 x \cdot \nabla_{x} z+d z\right)
$$

so that $z$ solution of A.1 verifies

$$
\partial_{t} z+S z+A z=g \text { in }(0, T) \times \Omega .
$$

Note that $S$ and $A$ respectively correspond to the symmetric and skew-symmetric parts of the operator in A.1.

We then consider

$$
D(t):=\int_{\Omega}\left(\left|\nabla_{x} z(t, x)\right|^{2}-\frac{L^{2}}{4 t^{2}}|z(t, x)|^{2}\right) d x=\int_{\Omega}(S z)(t, x) z(t, x) d x .
$$


A direct calculation shows that

$$
\begin{aligned}
D^{\prime}(t) & =\frac{L^{2}}{2 t^{3}} \int_{\Omega}|z|^{2} d x+2 \int_{\Omega} S z \partial_{t} z d x \\
& =\frac{L^{2}}{2 t^{3}} \int_{\Omega}|z|^{2} d x-2 \int_{\Omega}|S z|^{2} d x-2 \int_{\Omega} S z A z d x+2 \int_{\Omega} S z g d x .
\end{aligned}
$$

Furthermore, as $A$ is a skew-symmetric operator, we have

$$
-2 \int_{\Omega} S z A z d x=2 \int_{\Omega} \Delta_{x} z A z d x=\frac{1}{t} \int_{\Omega} \Delta_{x} z\left(2 x \cdot \nabla_{x} z+d z\right) d x .
$$

On one hand, we obviously have

$$
\int_{\Omega} \Delta_{x} z d z d x=-d \int_{\Omega}\left|\nabla_{x} z\right|^{2} d x
$$

On the other hand, we note that

$$
\begin{aligned}
\int_{\Omega} \Delta_{x} z 2 x \cdot \nabla_{x} z d x & =2 \int_{\partial \Omega}\left(\nabla_{x} z \cdot \nu\right)\left(x \cdot \nabla_{x} z\right) d s(x)-2 \int_{\Omega} \nabla_{x} z \cdot \nabla_{x}\left(x \cdot \nabla_{x} z\right) d x \\
& =2 \int_{\partial \Omega}(x \cdot \nu)\left|\nabla_{x} z \cdot \nu\right|^{2} d s(x)-2 \int_{\Omega} \nabla_{x} z \cdot \nabla_{x}\left(x \cdot \nabla_{x} z\right) d x .
\end{aligned}
$$

Here, we have used that as $z=0$ on $\partial \Omega, \nabla_{x} z=\left(\nabla_{x} z \cdot \nu\right) \nu$ on $\partial \Omega$. As

$$
\nabla_{x} z \cdot \nabla_{x}\left(x \cdot \nabla_{x} z\right)=\left|\nabla_{x} z\right|^{2}+\frac{x}{2} \cdot \nabla_{x}\left(\left|\nabla_{x} z\right|^{2}\right),
$$

we have

$$
\begin{aligned}
\int_{\Omega} \nabla_{x} z \cdot \nabla_{x}\left(x \cdot \nabla_{x} z\right) d x & =\int_{\Omega}\left|\nabla_{x} z\right|^{2} d x+\int_{\Omega} \frac{x}{2} \cdot \nabla_{x}\left(\left|\nabla_{x} z\right|^{2}\right) d x \\
& =\int_{\Omega}\left|\nabla_{x} z\right|^{2} d x+\frac{1}{2} \int_{\partial \Omega}(x \cdot \nu)\left|\nabla_{x} z\right|^{2} d s(x)-\frac{d}{2} \int_{\Omega}\left|\nabla_{x} z\right|^{2} d x \\
& =\int_{\Omega}\left|\nabla_{x} z\right|^{2} d x+\frac{1}{2} \int_{\partial \Omega}(x \cdot \nu)\left|\nabla_{x} z \cdot \nu\right|^{2} d s(x)-\frac{d}{2} \int_{\Omega}\left|\nabla_{x} z\right|^{2} d x .
\end{aligned}
$$

Gathering the above computations, we get that

$$
\begin{aligned}
D^{\prime}(t)+2 \int_{\Omega}|S z|^{2} d x & =\frac{L^{2}}{2 t^{3}} \int_{\Omega}|z|^{2} d x-\frac{2}{t} \int_{\Omega}\left|\nabla_{x} z\right|^{2} d x+\frac{1}{t} \int_{\partial \Omega}(x \cdot \nu)\left|\nabla_{x} z \cdot \nu\right|^{2} d s(x)+2 \int_{\Omega} S z g d x \\
& \leqslant-\frac{2}{t} D(t)+\frac{1}{t} \int_{\partial \Omega}(x \cdot \nu)\left|\nabla_{x} z \cdot \nu\right|^{2} d s(x)+\int_{\Omega}|S z|^{2} d x+\int_{\Omega}|g|^{2} d x
\end{aligned}
$$

which implies in particular

$$
\left(t^{2} D(t)\right)^{\prime} \leqslant t \int_{\Gamma_{+}}(x \cdot \nu)\left|\nabla_{x} z \cdot \nu\right|^{2} d s(x)+t^{2} \int_{\Omega}|g|^{2} d x .
$$

Using the assumption on $z$ in A.1 (3,4), one easily checks $\lim _{t \rightarrow 0} t^{2} D(t)=0$, hence we can integrate A.4 between 0 and $T$, which gives $(\overline{\mathrm{A} .3})$, as $|(x \cdot \nu)| \leqslant L$ for all $x \in \bar{\Omega}$.

\section{References}

[1] C. Bardos, G. Lebeau, and J. Rauch. Un exemple d'utilisation des notions de propagation pour le contrôle et la stabilisation de problèmes hyperboliques. Rend. Sem. Mat. Univ. Politec. Torino, (Special Issue):11-31 (1989), 1988. Nonlinear hyperbolic equations in applied sciences.

[2] C. Bardos, G. Lebeau, and J. Rauch. Sharp sufficient conditions for the observation, control and stabilization of waves from the boundary. SIAM J. Control and Optim., 30(5):1024-1065, 1992. 
[3] N. Burq and P. Gérard. Condition nécessaire et suffisante pour la contrôlabilité exacte des ondes. $C$. R. Acad. Sci. Paris Sér. I Math., 325(7):749-752, 1997.

[4] J.-M. Coron and S. Guerrero. Singular optimal control: a linear 1-D parabolic-hyperbolic example. Asymptot. Anal., 44(3-4):237-257, 2005.

[5] J.-M. Coron and H.-M. Nguyen. Null Controllability and Finite Time Stabilization for the Heat Equations with Variable Coefficients in Space in One Dimension via Backstepping Approach. Arch. Ration. Mech. Anal., 225(3):993-1023, 2017.

[6] J. Dardé and S. Ervedoza. On the reachable set for the one-dimensional heat equation.

[7] B. Dehman and S. Ervedoza. Observability estimates for the wave equation with rough coefficients. $C$. R. Math. Acad. Sci. Paris, 355(5):499-514, 2017.

[8] D. Dos Santos Ferreira, C. E. Kenig, M. Salo, and G. Uhlmann. Limiting Carleman weights and anisotropic inverse problems. Invent. Math., 178(1):119-171, 2009.

[9] J. W. Eaton, D. Bateman, S. Hauberg, and R. Wehbring. GNU Octave version 3.8.1 manual: a high-level interactive language for numerical computations, volume ISBN 1441413006. CreateSpace Independent Publishing Platform, http://www.gnu.org/software/octave/doc/interpreter/, 2014.

[10] Ju. V. Egorov. Some problems in the theory of optimal control. Ž. Vyčisl. Mat. i Mat. Fiz., 3:887-904, 1963.

[11] F. Fanelli and E. Zuazua. Weak observability estimates for 1-D wave equations with rough coefficients. Ann. Inst. H. Poincaré Anal. Non Linéaire, 32(2):245-277, 2015.

[12] H. O. Fattorini and D. L. Russell. Exact controllability theorems for linear parabolic equations in one space dimension. Arch. Rational Mech. Anal., 43:272-292, 1971.

[13] W. H. J. Fuchs. On the eigenvalues of an integral equation arising in the theory of band-limited signals. J. Math. Anal. Appl., 9:317-330, 1964.

[14] A. V. Fursikov and O. Y. Imanuvilov. Controllability of evolution equations, volume 34 of Lecture Notes Series. Seoul National University Research Institute of Mathematics Global Analysis Research Center, Seoul, 1996.

[15] D. Gilbarg and N. S. Trudinger. Elliptic partial differential equations of second order. Classics in Mathematics. Springer-Verlag, Berlin, 2001. Reprint of the 1998 edition.

[16] H. Gimperlein and A. Waters. A deterministic optimal design problem for the heat equation. SIAM J. Control Optim., 55(1):51-69, 2017.

[17] O. Glass. A complex-analytic approach to the problem of uniform controllability of a transport equation in the vanishing viscosity limit. J. Funct. Anal., 258(3):852-868, 2010.

[18] I. S. Gradshteyn and I. M. Ryzhik. Table of integrals, series, and products. Elsevier/Academic Press, Amsterdam, seventh edition, 2007. Translated from the Russian, Translation edited and with a preface by Alan Jeffrey and Daniel Zwillinger, With one CD-ROM (Windows, Macintosh and UNIX).

[19] E. N. Güichal. A lower bound of the norm of the control operator for the heat equation. J. Math. Anal. Appl., 110(2):519-527, 1985.

[20] F. Hecht. New development in freefem++. J. Numer. Math., 20(3-4):251-265, 2012.

[21] P. Henrici. Applied and computational complex analysis. Vol. 1. Wiley Classics Library. John Wiley \& Sons, Inc., New York, 1988. Power series-integration-conformal mapping-location of zeros, Reprint of the 1974 original, A Wiley-Interscience Publication.

[22] L. F. Ho. Observabilité frontière de l'équation des ondes. C. R. Acad. Sci. Paris Sér. I Math., 302(12):443-446, 1986.

[23] L. Hörmander. The analysis of linear partial differential operators. III, volume 274 of Grundlehren der Mathematischen Wissenschaften. Springer-Verlag, Berlin, 1985. Pseudodifferential operators.

[24] H. J. Landau and H. O. Pollak. Prolate spheroidal wave functions, Fourier analysis and uncertainty. II. Bell System Tech. J., 40:65-84, 1961. 
[25] G. Lebeau and L. Robbiano. Contrôle exact de l'équation de la chaleur. Comm. Partial Differential Equations, 20(1-2):335-356, 1995.

[26] N. N. Lebedev. Special functions and their applications. Dover Publications, Inc., New York, 1972. Revised edition, translated from the Russian and edited by Richard A. Silverman, Unabridged and corrected republication.

[27] J.-L. Lions. Contrôlabilité exacte, Stabilisation et Perturbations de Systèmes Distribués. Tome 1. Contrôlabilité exacte, volume RMA 8. Masson, 1988.

[28] P. Lissy. A link between the cost of fast controls for the 1-D heat equation and the uniform controllability of a 1-D transport-diffusion equation. C. R. Math. Acad. Sci. Paris, 350(11-12):591-595, 2012.

[29] P. Lissy. Explicit lower bounds for the cost of fast controls for some 1-D parabolic or dispersive equations, and a new lower bound concerning the uniform controllability of the 1-D transport diffusion equation. Journal of Differential Equations, 259(10):5331-5352, 2015.

[30] P. Martin, L. Rosier, and P. Rouchon. Null controllability of the heat equation using flatness. Automatica J. IFAC, 50(12):3067-3076, 2014.

[31] P. Martin, L. Rosier, and P. Rouchon. On the reachable sets for the boundary control of the heat equation. Applied Mathematics Research eXpress, 2016.

[32] L. Miller. Geometric bounds on the growth rate of null-controllability cost for the heat equation in small time. J. Differential Equations, 204(1):202-226, 2004.

[33] L. Miller. On the null-controllability of the heat equation in unbounded domains. Bull. Sci. Math., 129(2):175-185, 2005.

[34] L. Miller. The control transmutation method and the cost of fast controls. SIAM J. Control Optim., 45(2):762-772 (electronic), 2006.

[35] M. H. Protter and H. F. Weinberger. Maximum principles in differential equations. Springer-Verlag, New York, 1984. Corrected reprint of the 1967 original.

[36] T. I. Seidman. Two results on exact boundary control of parabolic equations. Appl. Math. Optim., 11(2):145-152, 1984.

[37] G. Tenenbaum and M. Tucsnak. New blow-up rates for fast controls of Schrödinger and heat equations. J. Differential Equations, 243(1):70-100, 2007. 